Research Article

\title{
Free Vibration and Static Bending Analysis of Piezoelectric Functionally Graded Material Plates Resting on One Area of Two-Parameter Elastic Foundation
}

\author{
Hong Nguyen Thi \\ Faculty of Mechanical Engineering, Thuyloi University, 175 Tay Son, Dong Da, Hanoi, Vietnam \\ Correspondence should be addressed to Hong Nguyen Thi; hong_hh@tlu.edu.vn
}

Received 10 August 2020; Revised 19 October 2020; Accepted 8 November 2020; Published 25 November 2020

Academic Editor: Nerio Tullini

Copyright (C) 2020 Hong Nguyen Thi. This is an open access article distributed under the Creative Commons Attribution License, which permits unrestricted use, distribution, and reproduction in any medium, provided the original work is properly cited.

\begin{abstract}
Free vibration and static bending analysis of piezoelectric functionally graded material plates resting on one area of the twoparameter elastic foundation is firstly investigated in this paper. The third-order shear deformation theory of Reddy and 8-node plate elements are employed to derive the finite element formulations of the structures; this theory does not need any shear correction factors; however, the mechanical response of the structure is described exactly. Verification problems are performed to evaluate the accuracy of the proposed theory and mathematical model. A wide range of parameter study is investigated to figure out the effect of geometrical, physical, and material properties such as the plate dimension, volume fraction index, piezoelectric effect, elastic foundation coefficients, and the square size of the area of the foundation on the free vibration and static bending of piezoelectric functionally graded material plates. These numerical results of this work aim to contribute to scientific knowledge of these smart structures in engineering practice.
\end{abstract}

\section{Introduction}

Nowadays, due to the development of the science of technology, a huge range of smart materials such as functionally graded materials, shape memory alloys, shape memory polymers, magnetostrictive materials, and piezoelectric materials has been considered and applied to engineering practice, in which piezoelectric materials with mainly twocomponent actuators and sensors are employed and performed in a wide range of engineering structures [1-5]. Normally, one smart structure is commonly made of laminated composites from ceramic proportions. Therefore, at the surfaces among such layers, due to discontinuities of different materials, the stress concentrations usually occur; thus, this is one of the main reasons deriving the failures of the laminated composite structures. However, this issue can be easily overcome by taking advantage of functionally graded piezoelectric materials (FGPM). In the last few decades, considering the mechanical behavior of piezoelectric FGM structures has become more irresistible. To confirm this knowledge, numerous published scientific papers relating to piezoelectric analyses for FGPM plates can be found in [6-16]. As a result, in this work, we do not need to talk about the basic information of FGM structures as several types of FGM materials such as power-law functionally graded materials (P-FGM), sigmoid law functionally graded materials (S-FGM), and exponential law functionally graded materials (E-FGM) were presented in detail in $[17,18]$. The mechanical performances of these FGM structures are also investigated in the following papers. Tinh et al. [19] studied mechanical behaviors of heated functionally graded plates in the high-temperature environment by using the finite element method and a new third-order shear deformation plate theory. Nguyen and his coworkers [20] investigated the mechanical buckling of stiffened functionally graded material (FGM) plates. A wide range of studied parameters was carried out such as the effects of material distribution, the thickness-to-width ratio, and stiffener parameters on the buckling characteristics of the stiffened FGM plates. Nam et al. [21] used the finite element method and the phase-field 
model to consider the buckling behavior of stiffened functionally graded (FG) plate with cracks. Besides, the interactions of the mechanical structures with the elastic foundations have been taken into account in many investigations of scientists worldwide. For example, to describe the behavior between the plate and foundation, different types of mathematical elastic foundation models have been introduced $[22,23]$. The simplest one is Winkler, usually called one-parameter elastic foundations, which assumes the foundation as a series of dependent springs without coupling impacts among each other [24]. Then, this model is developed by Pasternak taking into account a shear spring component to model the interactions among the separated springs in Winkler's elastic foundation [25-30]. Besides, substantial investigations depended on classical plate theory [31, 32], first-order shear deformation theory [24, 33-35], higher-order shear deformation [36], and three-dimensional elasticity theory (3D theory) [32, 37-39]. Zenkour and coworkers [40] introduced the bending static analysis of fiber-reinforced viscoelastic composite plate resting on the two-parameter elastic foundation of Pasternak. Several explorations concerning different mechanical responses of FG plates using HSDT and resting on elastic foundations have been arisen [41-43]. Abderrahmane et al. [44] used the analytical method to study the dynamic behaviors of the FG Sandwich plate resting on an elastic foundation based on refined shear deformation theory. Abdallah et al. [45] employed a cubic shear deformation theory and the exact solution to examine the bending response of FG porous plates. Mohamed et al. [46] used a new innovative 3-unknown HSDT to research buckling and free vibration of FG Sandwich plates resting on elastic foundations. Mokhtar et al. [47] employed a four-variable quasi-3D HSDT to give out the exact solution for the static and vibration problems of thick advanced composite plates. Sara and her coworkers [48] studied the buckling response of FG Sandwich plates resting on elastic foundation based on a novel four-unknown integral model. Salah et al. [49] also studied buckling of FG Sandwich plates resting on elastic foundation subjected to hygro-thermo-mechanical loads. Mohammed et al. [50] investigated the effects of the boundary conditions on the bending and free vibration behavior of FGM Sandwich plates using a four-unknown refined integral plate theory.

There are many approaches to calculating the static bending, free vibration, and dynamic problems of FGM plates such as the classical plate theory, first-order shear deformation theory (FSDT), and higher-order shear deformation theories. The classical plate theory [51] is suitable for thin plates, and it does not take into account the effect of the shear force. Although the FSDT takes into account the effect of shear force, it cannot describe the exact distribution of shear stress according to the plate thickness; besides, the calculation needs to use the shear correction factor. The third-order shear deformation theory $[8,52]$ presents exactly the distribution of shear stress in the thickness direction and does not use any shear correction factors. Notwithstanding, although the calculation is more complicated than the classical plate theory and the FSDT, Reddy's plate theory is suitable for solving the complicated structures such as piezoelectric homogeneous and heterogeneous structures. Moreover, some piezoelectric plates are only partially leaning on other structures, possibly modeled as one area elastic foundation, which has not been specified in the existing studies. It is also because the plate only rests on one area elastic foundation, so the mechanical behavior of this plate will be different from the case of a fully elastic foundation. The modeling and calculation of these structures using the higher-order shear deformation theory of Reddy are interesting problems on how to use them most effectively in engineering practice. This is also the main purpose of this work. Besides, the 8-node plate element is used to present high confidence through comparison with previously published literature.

The rest of this paper is as follows. Section 2 introduces shortly piezoelectric functionally graded plates. Finite element formulations for free vibration and static bending of piezoelectric FGM plates are presented in detail in Section 3. Section 4 performs verification problems, numerical results, and discussions. Some bold conclusions are summed up in Section 5.

\section{Piezoelectric Functionally Graded Plates}

Considering that a piezoelectric FGM plate with the core layer is FGM, the other outer ones attacked the piezoelectric components, called actuator and sensor layers. One area of the plate rests on the elastic foundation with the length $d_{e}$ and two stiffness coefficients $k_{w}$ and $k_{s}$ (Figure 1).

Assuming that the core layer is the FGM made of ceramic and metal materials, the material properties of the material at the point with $z$ coordinate have the following expressions [52]:

$$
\left\{\begin{array}{l}
E(x)=E_{c} V_{c}+E_{m} V_{m}, \\
v(x)=v_{c} V_{c}+v_{m} V_{m} \\
\rho(x)=\rho_{c} V_{c}+\rho_{m} V_{m}
\end{array}\right.
$$

where $E_{i}, v_{i}, \rho_{i}$, and $V_{i}(i=c, m)$ are Young's modulus, Poisson's ratio, mass density, and the volume fraction of $i$-th material, $x$ and $y$ are lines of in-plane of midsurface, $z$ defines the normal axis, $c$ denotes ceramic, and $m$ denotes metal. The volume fractions of materials in this paper are given by [52]

$$
\left\{\begin{array}{l}
V_{c}=\left[1-\left(\frac{z}{h}+\frac{1}{2}\right)^{n}\right], \\
V_{m}=1-V_{c},
\end{array}\right.
$$



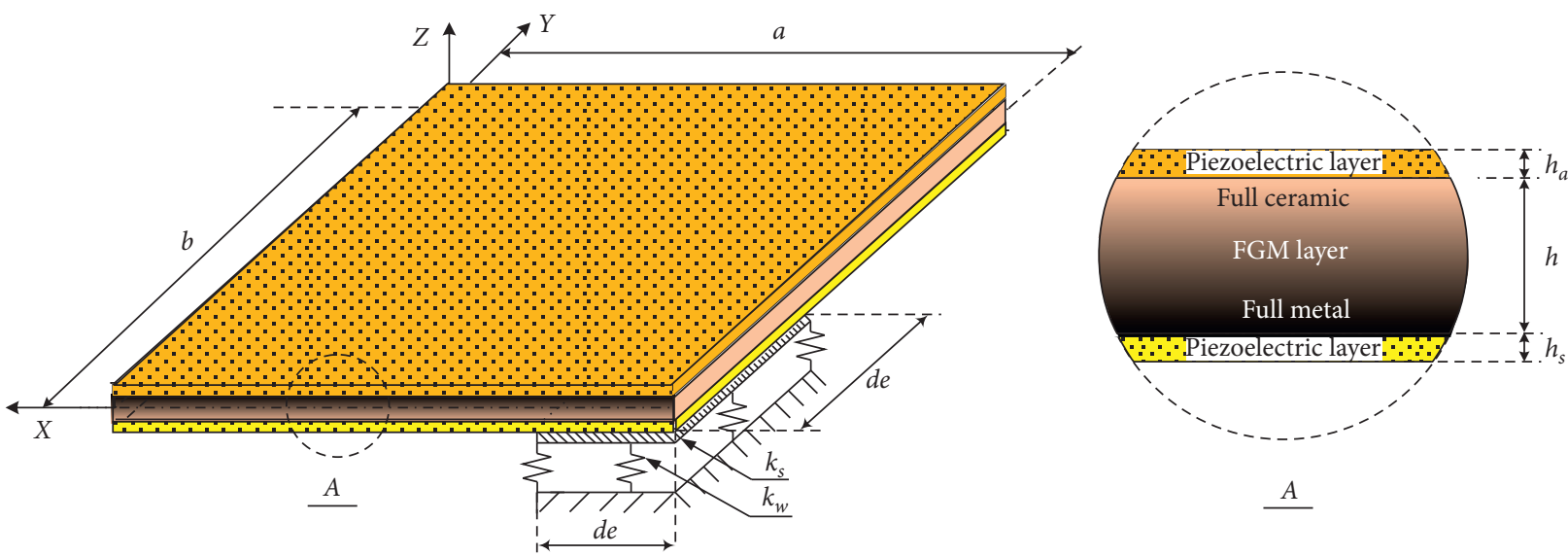

FIGURE 1: Schematic of a piezoelectric FGM plate resting on one area of the elastic foundation.

where $n$ is the material parameter with a nonnegative value.

\section{Finite Element Formulations for Free Vibration of Piezoelectric FGM Plates}

Many plate theories can be used to explore the mechanical behavior of the plate structures. In this work, the high-order shear deformation plate theory of Reddy is employed; therefore, the displacement field at any points within the plate is given as follows [52]:

$$
\left\{\begin{array}{l}
u(x, y, z)=u_{0}(x, y)+z \varphi_{x}(x, y)-\frac{4}{3 h^{2}} z^{3}\left(\varphi_{x}+\frac{\partial w_{0}}{\partial x}\right) \\
v(x, y, z)=v_{0}(x, y)+z \varphi_{y}(x, y)-\frac{4}{3 h^{2}} z^{3}\left(\varphi_{y}+\frac{\partial w_{0}}{\partial y}\right) \\
w(x, y, z)=w_{0}(x, y)
\end{array}\right.
$$

where $u_{0} ; v_{0} ; w_{0}$ are the displacements at a point $(x, y, z=0)$; $\varphi_{x}, \varphi_{y}$ are the transverse normal rotations in $x z$ - and $y z$ surfaces, respectively.

The strains can be written in detail as

$$
\left\{\begin{array}{l}
\varepsilon_{x}=\varepsilon_{x}^{0}+z \varepsilon_{x}^{1}+z^{3} \varepsilon_{x}^{3} \\
\varepsilon_{y}=\varepsilon_{y}^{0}+z \varepsilon_{y}^{1}+z^{3} \varepsilon_{y}^{3} \\
\gamma_{x y}=\gamma_{x y}^{0}+z \gamma_{x}^{1}+z^{3} \gamma_{x}^{3} \\
\gamma_{x z}=\gamma_{x z}^{0}+z^{2} \gamma_{x z}^{2} \\
\gamma_{y z}=\gamma_{y z}^{0}+z^{2} \gamma_{y z}^{2}
\end{array}\right.
$$

$$
\begin{aligned}
& \boldsymbol{\varepsilon}^{0}=\left\{\begin{array}{c}
\varepsilon_{x}^{0} \\
\varepsilon_{y}^{0} \\
\gamma_{x y}^{0}
\end{array}\right\}=\left\{\begin{array}{c}
\frac{\partial u_{0}}{\partial x} \\
\frac{\partial v_{0}}{\partial y} \\
\frac{\partial v_{0}}{\partial x}+\frac{\partial u_{0}}{\partial y}
\end{array}\right\} \\
& \boldsymbol{\varepsilon}_{x}^{1}=\left\{\begin{array}{c}
\varepsilon_{x}^{1} \\
\varepsilon_{y}^{1} \\
\gamma_{x y}^{1}
\end{array}\right\}=\left\{\begin{array}{c}
\frac{\partial \varphi_{x}}{\partial x} \\
\frac{\partial \varphi_{y}}{\partial y} \\
\frac{\partial \varphi_{x}}{\partial y}+\frac{\partial \varphi_{y}}{\partial x}
\end{array}\right\} \text {, } \\
& \boldsymbol{\varepsilon}_{x}^{3}=\left\{\begin{array}{c}
\varepsilon_{x}^{3} \\
\varepsilon_{y}^{3} \\
\gamma_{x y}^{3}
\end{array}\right\}=\frac{-4}{3 h^{2}}\left\{\begin{array}{c}
\frac{\partial \varphi_{x}}{\partial x}+\frac{\partial^{2} w_{0}}{\partial x^{2}} \\
\frac{\partial \varphi_{y}}{\partial y}+\frac{\partial^{2} w_{0}}{\partial y^{2}} \\
\frac{\partial \varphi_{x}}{\partial y}+\frac{\partial \varphi_{y}}{\partial x}+\frac{2 \partial^{2} w_{0}}{\partial x \partial y}
\end{array}\right\}, \\
& \gamma^{0}=\left\{\begin{array}{l}
\gamma_{x z}^{0} \\
\gamma_{y z}^{0}
\end{array}\right\}=\left\{\begin{array}{l}
\varphi_{x}+\frac{\partial w_{0}}{\partial x} \\
\varphi_{y}+\frac{\partial w_{0}}{\partial y}
\end{array}\right\} \\
& \gamma^{2}=\left\{\begin{array}{c}
\gamma_{x z}^{2} \\
\gamma_{y z}^{2}
\end{array}\right\}=\frac{-4}{h^{2}}\left\{\begin{array}{c}
\varphi_{x}+\frac{\partial w_{0}}{\partial x} \\
\varphi_{y}+\frac{\partial w_{0}}{\partial y}
\end{array}\right\}
\end{aligned}
$$

Equation (4) can be written in a short form as follows: 
The linear piezoelectric coupling between the elastic field and the electric field can be expressed by the direct and the converse piezoelectric equations; the relationship between stresses and strains are written by $[53,54]$

$$
\left\{\begin{array}{l}
\boldsymbol{\sigma}=\mathbf{D}_{b}\left(\boldsymbol{\varepsilon}^{0}+z \boldsymbol{\varepsilon}^{1}+z^{3} \varepsilon^{3}\right)-\mathbf{e}^{T} \mathbf{E}_{v} \\
\boldsymbol{\tau}=\mathbf{D}_{s}\left(\gamma^{0}+z^{2} \gamma^{2}\right), \\
\mathbf{D}=\mathbf{e}\left(\boldsymbol{\varepsilon}^{0}+z \boldsymbol{\varepsilon}^{1}+z^{3} \varepsilon^{3}\right)+\mathbf{p} \mathbf{E}_{v}
\end{array}\right.
$$

where

$$
\begin{aligned}
\boldsymbol{\sigma} & =\left\{\begin{array}{c}
\boldsymbol{\sigma}_{x} \\
\boldsymbol{\sigma}_{y} \\
\boldsymbol{\tau}_{x y}
\end{array}\right\} ; \\
\boldsymbol{\tau} & =\left\{\begin{array}{c}
\boldsymbol{\tau}_{x z} \\
\boldsymbol{\tau}_{y z}
\end{array}\right\}, \\
\mathbf{D}_{b} & =\frac{E(z)}{1-v^{2}(z)}\left[\begin{array}{ccc}
1 & v(z) & 0 \\
\nu(z) & 1 & 0 \\
0 & 0 & \frac{1-\nu(z)}{2}
\end{array}\right] ; \\
\mathbf{D}_{s} & =\frac{E(z)}{2(1+v(z))}\left[\begin{array}{ll}
1 & 0 \\
0 & 1
\end{array}\right] .
\end{aligned}
$$

(i) $\mathbf{E}_{v}$ is the electric field, which can be defined through the variation of voltage as follows [55]:

$$
\mathbf{E}_{v}=-\nabla \phi=\left\{\begin{array}{c}
0 \\
0 \\
E^{z}
\end{array}\right\},
$$

where the effect of the electric field is considered only in the thickness direction of the plate; it is symbolized as $E^{z}$ and $E^{z}$ is calculated through the voltage and the thickness as

$$
E^{z}=\frac{-\phi}{h_{p}}
$$

in which $h_{p}$ is the thickness of actuator and sensor layers, $h_{p}=h_{a}$ and $h_{p}=h_{s}$.

(ii) $\mathbf{D}$ is the electric displacement vector.

(iii) e denotes the piezoelectric stress coefficients with the following particular expression:

$$
\mathbf{e}=\left[\begin{array}{ccc}
0 & 0 & 0 \\
0 & 0 & 0 \\
e_{31} & e_{32} & 0
\end{array}\right] .
$$

(iv) $\mathbf{p}$ is the dielectric tensor at constant mechanical strain with the following expression:

$$
\mathbf{p}=\left[\begin{array}{ccc}
p_{11} & 0 & 0 \\
0 & p_{22} & 0 \\
0 & 0 & p_{33}
\end{array}\right] .
$$

This work uses an 8-node element, where each node has 5 degrees of freedom:

$$
\begin{aligned}
& u_{0}=\sum_{j=1}^{8} N_{j}(\xi, \eta) \cdot u_{j} ; \\
& v_{0}=\sum_{j=1}^{8} N_{j}(\xi, \eta) \cdot v_{j} ; \\
& w_{0}=\sum_{j=1}^{8} N_{j}(\xi, \eta) \cdot w_{0 j}, \\
& \varphi_{x}=\sum_{j=1}^{8} N_{j}(\xi, \eta) \cdot \varphi_{x j} ; \\
& \varphi_{y}=\sum_{j=1}^{8} N_{j}(\xi, \eta) \cdot \varphi_{y j},
\end{aligned}
$$

where $N_{j}$ is the Lagrangian interpolation function of an 8node element and $\xi, \eta$ are the natural coordinates.

So the strains relate to element displacement vector as follows: 


$$
\left\{\begin{array}{c}
\boldsymbol{\varepsilon}^{0}=\mathbf{B}_{0} \mathbf{q}_{e} \\
\boldsymbol{\varepsilon}^{1}=\mathbf{B}_{1} \mathbf{q}_{e} \\
\boldsymbol{\varepsilon}^{3}=\mathbf{B}_{3} \mathbf{q}_{e} \\
\gamma^{0}=\mathbf{B}_{0 \gamma} \mathbf{q}_{e} \\
\gamma^{2}=\mathbf{B}_{2 \gamma} \mathbf{q}_{e}
\end{array}\right.
$$

At this time, the electric vector of actuator and sensor layers is calculated through two degrees of freedom of actuator and sensor layers as follows:

$$
\mathbf{E}_{v}=-\left[\begin{array}{c}
0,0, \frac{1}{h_{a}}, 0,0,0 \\
0,0,0,0,0, \frac{1}{h_{s}}
\end{array}\right]^{T}\left\{\begin{array}{l}
\phi_{a} \\
\phi_{s}
\end{array}\right\}=-\left[\begin{array}{cc}
\mathbf{B}_{\phi}^{a} & 0 \\
0 & \mathbf{B}_{\phi}^{s}
\end{array}\right]\left\{\begin{array}{l}
\phi_{a} \\
\phi_{s}
\end{array}\right\},
$$

with

$$
\mathbf{B}_{0}=\sum_{j=1}^{8}\left[\begin{array}{ccccc}
\frac{\partial N_{j}}{\partial x} & 0 & 0 & 0 & 0 \\
0 & \frac{\partial N_{j}}{\partial y} & 0 & 0 & 0 \\
\frac{\partial N_{j}}{\partial y} \frac{\partial N_{j}}{\partial x} & 0 & 0 & 0
\end{array}\right], \quad \mathbf{B}_{\phi}^{a}=\left[0,0, \frac{1}{h_{a}}\right]^{T} ;
$$$$
\mathbf{B}_{1}=\sum_{j=1}^{8}\left[\begin{array}{ccccc}
0 & 0 & 0 & \frac{\partial N_{j}}{\partial x} & 0 \\
0 & 0 & 0 & 0 & \frac{\partial N_{j}}{\partial y} \\
0 & 0 & 0 & \frac{\partial N_{j}}{\partial y} & \frac{\partial N_{j}}{\partial x}
\end{array}\right],
$$$$
\mathbf{B}_{3}=\frac{-4}{3 h^{2}} \sum_{j=1}^{8}\left[\begin{array}{ccccc}
0 & 0 & \frac{\partial^{2} N_{j}}{\partial x^{2}} & \frac{\partial N_{j}}{\partial x} & 0 \\
0 & 0 & \frac{\partial^{2} N_{j}}{\partial y^{2}} & 0 & \frac{\partial N_{j}}{\partial y} \\
0 & 0 & 2 \frac{\partial^{2} N_{j}}{\partial x \partial y} & \frac{\partial N_{j}}{\partial y} & \frac{\partial N_{j}}{\partial x}
\end{array}\right],
$$$$
\mathbf{B}_{0 \gamma}=\sum_{j=1}^{8}\left[\begin{array}{ccccc}
0 & 0 & \frac{\partial N_{j}}{\partial x} & 0 & N_{j} \\
0 & 0 & \frac{\partial N_{j}}{\partial y} & N_{j} & 0
\end{array}\right] \text {; }
$$$$
\mathbf{B}_{2 \gamma}=\frac{-4}{h^{2}} \sum_{j=1}^{8}\left[\begin{array}{ccccc}
0 & 0 & \frac{\partial N_{j}}{\partial x} & 0 & N_{j} \\
0 & 0 & \frac{\partial N_{j}}{\partial y} & N_{j} & 0
\end{array}\right] \text {. }
$$

where $h_{a}$ and $h_{s}$ are the thicknesses of the actuator and sensor layers, respectively.

After integrating from $-h / 2$ to $h / 2$, the normal force, bending moments, high-order bending moments, shear force, and high-order shear force can be expressed as

$$
\left\{\begin{array}{c}
\mathbf{N} \\
\mathbf{M} \\
\widehat{M} \\
\mathbf{Q} \\
\widehat{Q}
\end{array}\right\}=\left[\begin{array}{ccccc}
\mathbf{A} & \mathbf{B} & \mathbf{E} & \mathbf{0} & \mathbf{0} \\
\mathbf{B} & \mathbf{D} & \mathbf{F} & \mathbf{0} & \mathbf{0} \\
\mathbf{E} & \mathbf{F} & \mathbf{H} & \mathbf{0} & \mathbf{0} \\
\mathbf{0} & \mathbf{0} & \mathbf{0} & \bar{A} & \bar{B} \\
\mathbf{0} & \mathbf{0} & \mathbf{0} & \bar{B} & \bar{D}
\end{array}\right]\left\{\begin{array}{c}
\boldsymbol{\varepsilon}^{0} \\
\boldsymbol{\varepsilon}^{1} \\
\boldsymbol{\varepsilon}^{3} \\
\boldsymbol{\gamma}^{0} \\
\boldsymbol{\gamma}^{2}
\end{array}\right\}
$$

where

$$
\begin{aligned}
& \left\{\begin{array}{c}
\mathbf{A} \\
\mathbf{B} \\
\mathbf{D} \\
\mathbf{E} \\
\mathbf{F} \\
\mathbf{H}
\end{array}\right\}=\int_{-(h / 2)}^{h / 2} \mathbf{D}_{b}\left\{\begin{array}{c}
1 \\
z \\
z^{2} \\
z^{3} \\
z^{4} \\
z^{6}
\end{array}\right\} \mathrm{d} z ; \\
& \left\{\begin{array}{c}
\bar{A} \\
\bar{B} \\
\bar{D}
\end{array}\right\}=\int_{-(h / 2)}^{h / 2} \mathbf{D}_{s}\left\{\begin{array}{c}
1 \\
z^{2} \\
z^{4}
\end{array}\right\} \mathrm{d} z \text {. }
\end{aligned}
$$

The total strain energy of the piezoelectric FGM plate can be given by 


$$
\begin{aligned}
& \prod_{e}=\frac{1}{2} \int_{V_{e}}\left(\begin{array}{c}
\left\{\begin{array}{ccc}
\boldsymbol{\varepsilon}^{0} & \boldsymbol{\varepsilon}^{1} & \boldsymbol{\varepsilon}^{3}
\end{array}\right\}^{T} \sigma+\left\{\begin{array}{cc}
\boldsymbol{\gamma}^{0} & \boldsymbol{\gamma}^{2}
\end{array}\right\}^{T} \boldsymbol{\tau}-\mathbf{D}^{T} \mathbf{E}_{v} \\
k_{w} w^{2}+k_{s}\left(\left(\frac{\partial w}{\partial x}\right)^{T}\left(\frac{\partial w}{\partial x}\right)+\left(\frac{\partial w}{\partial y}\right)^{T}\left(\frac{\partial w}{\partial y}\right)\right)
\end{array}\right) \mathrm{d} \mathrm{d} z-\int_{S_{e}} \mathbf{u}^{T} \widetilde{F} \mathrm{~d} S-\int_{S_{e}} \mathbf{E}_{v}^{T} V \mathrm{~d} S \\
& =\mathbf{q}_{e}^{T} \frac{1}{2} \int_{S_{e}}\left(\mathbf{B}_{0}^{T} \mathbf{A B}_{0}+\mathbf{B}_{0}^{T} \mathbf{B B}_{1}+\mathbf{B}_{1}^{T} \mathbf{E B}_{3}+\mathbf{B}_{1}^{T} \mathbf{B B}_{0}+\mathbf{B}_{1}^{T} \mathbf{D} \mathbf{B}_{1}+\mathbf{B}_{0}^{T} \mathbf{E B}_{3}+\mathbf{B}_{3}^{T} \mathbf{E B}_{0}+\mathbf{B}_{1}^{T} \mathbf{F B}_{3}+\mathbf{B}_{3}^{T} \mathbf{E B}_{1}+\mathbf{B}_{3}^{T} \mathbf{F B}_{1}\right. \\
& \left.+\mathbf{B}_{3}^{T} \mathbf{H} \mathbf{B}_{3}+\mathbf{B}_{0 \gamma}^{T} \bar{A} \mathbf{B}_{0 \gamma}+\mathbf{B}_{0 \gamma}^{T} \bar{B} \mathbf{B}_{2 \gamma}+\mathbf{B}_{2 \gamma}^{T} \bar{B} \mathbf{B}_{0 \gamma}+\mathbf{B}_{2 \gamma}^{T} \bar{B} \mathbf{B}_{2 \gamma}+k_{w} \mathbf{N}_{w}^{T} \mathbf{N}_{w}+k_{s}\left(\left(\frac{\partial \mathbf{N}_{w}}{\partial x}\right)^{T}\left(\frac{\partial \mathbf{N}_{w}}{\partial x}\right)+\left(\frac{\partial \mathbf{N}_{w}}{\partial y}\right)^{T}\left(\frac{\partial \mathbf{N}_{w}}{\partial y}\right)\right)\right) \mathrm{d} S \mathbf{q}_{e} \\
& -\mathbf{q}_{e}^{T} \frac{1}{2} \int_{S_{e}}\left(-\mathbf{B}_{0}^{T} \mathbf{A} e^{T} \mathbf{B}_{\phi a}-\mathbf{B}_{1}^{T} \mathbf{B e}^{T} \mathbf{B}_{\phi a}-\mathbf{B}_{3}^{T} \mathbf{E e}^{T} \mathbf{B}_{\phi a}\right) \mathrm{d} S \phi_{e a} \\
& -\mathbf{q}_{e}^{T} \frac{1}{2} \int_{S_{e}}\left(-\mathbf{B}_{0}^{T} \mathbf{A} e^{T} \mathbf{B}_{\phi s}-\mathbf{B}_{1}^{T} \mathbf{B e}^{T} \mathbf{B}_{\phi s}-\mathbf{B}_{3}^{T} \mathbf{E e}^{T} \mathbf{B}_{\phi s}\right) \mathrm{d} S \phi_{e s} \\
& -\phi_{e a}^{T} \frac{1}{2} \int_{S_{e}}\left(-\mathbf{B}_{\phi a}^{T} \mathbf{e} \mathbf{A} \mathbf{B}_{0}-\mathbf{B}_{\phi a}^{T} \mathbf{e} \mathbf{B} \mathbf{B}_{1}-\mathbf{B}_{\phi a}^{T} \mathbf{e E B _ { 3 }}\right) \mathrm{d} S \mathbf{q}_{e} \\
& -\phi_{e s}^{T} \frac{1}{2} \int_{S_{e}}\left(-\mathbf{B}_{\phi s}^{T} \mathbf{e} \mathbf{A} \mathbf{B}_{0}-\mathbf{B}_{\phi s}^{T} \mathbf{e B B}_{1}-\mathbf{B}_{\phi s}^{T} \mathbf{e E B}_{3}\right) \mathrm{d} S \mathbf{q}_{e} \\
& -\frac{1}{2} \phi_{e a}^{T} \int_{S_{e}} \mathbf{B}_{\phi a}^{T} \mathbf{p} \mathbf{B}_{\phi a}^{T} \mathrm{~d} S \phi_{e a}-\frac{1}{2} \phi_{e s}^{T} \int_{S_{e}} \mathbf{B}_{\phi s}^{T} \mathbf{p} \mathbf{B}_{\phi s}^{T} \mathrm{~d} S \phi_{e s} \\
& -\mathbf{q}_{e}^{T} \int_{S_{e}} \mathbf{N}^{T} \widetilde{F} \mathrm{~d} S-\int_{S_{e}} \mathbf{E}_{v}^{T} V \mathrm{~d} S,
\end{aligned}
$$

where $\mathbf{N}_{w}=\sum_{j=1}^{8}\left[0,0, N_{j}, 0,0\right], \widetilde{F}$ is the surface loading, $V$ is the voltage applied to actuator and sensor layers, $\mathbf{q}_{e}$ is the nodal displacement vector, and $k_{w}$ and $k_{s}$ are the elastic foundation parameters. Equation (19) is expressed in matrix form as

$$
\prod_{e}=\frac{1}{2}\left(\mathbf{q}_{e}^{T} \mathbf{K}_{u u e} \mathbf{q}_{e}+\mathbf{q}_{e}^{T} \mathbf{K}_{u \phi e}^{a} \phi_{a e}+\mathbf{q}_{e}^{T} \mathbf{K}_{u \phi e}^{s} \phi_{s e}+\phi_{a e}^{T} \mathbf{K}_{\phi u e}^{a} \mathbf{q}_{e}+\phi_{s e}^{T} \mathbf{K}_{\phi u e}^{s} \mathbf{q}_{e}-\phi_{a e}^{T} \mathbf{K}_{\phi \phi e}^{a} \phi_{a e}-\phi_{s e}^{T} \mathbf{K}_{\phi \phi e}^{s} \phi_{s e}\right)-\mathbf{q}_{e}^{T} \widetilde{F}_{e}-\phi_{a e}^{T} \mathbf{F}_{q e}^{a}-\phi_{s e}^{T} \mathbf{F}_{q e}^{s},
$$

where the stiffness matrices are calculated as follows:

$$
\begin{aligned}
\underbrace{\mathbf{K}_{u u e}=}_{40 \times 40} & \int_{S_{e}}(\underbrace{\mathbf{B}_{0}^{T}}_{40 \times 3} \underbrace{\underset{\mathbf{A}}{\mathbf{B}_{0}}}_{3 \times 3} \underbrace{}_{3 \times 40}+\underbrace{\mathbf{B}_{0}^{T}}_{40 \times 3} \underbrace{\mathbf{B}}_{3 \times 3} \underbrace{\mathbf{B}_{1}}_{3 \times 40}+\underbrace{\mathbf{B}_{1}^{T}}_{40 \times 3} \underbrace{\mathbf{E}}_{3 \times 3} \underbrace{\mathbf{B}_{3}}_{3 \times 40}+\underbrace{\mathbf{B}_{1}^{T}}_{40 \times 3} \underbrace{\mathbf{B}}_{3 \times 3} \underbrace{\mathbf{B}_{0}}_{3 \times 40}+\underbrace{\mathbf{B}_{1}^{T}}_{40 \times 3} \underbrace{\mathbf{D}}_{3 \times 3} \underbrace{\mathbf{B}_{1}}_{3 \times 40}+\underbrace{\mathbf{B}_{0}^{T}}_{40 \times 3} \underbrace{\mathbf{E}}_{3 \times 3} \underbrace{\mathbf{B}_{3}}_{3 \times 40} \\
& +\underbrace{\mathbf{B}_{3}^{T}}_{40 \times 3} \underbrace{\mathbf{E}}_{3 \times 3} \underbrace{\mathbf{B}_{0}}_{3 \times 40}+\underbrace{\mathbf{B}_{1}^{T}}_{40 \times 3} \underbrace{\mathbf{E}}_{3 \times 3} \underbrace{\mathbf{B}_{3}}_{3 \times 40}+\underbrace{\mathbf{B}_{3}^{T}}_{40 \times 3} \underbrace{\mathbf{E}}_{3 \times 3} \underbrace{\mathbf{B}_{1}}_{3 \times 40}+\underbrace{\mathbf{B}_{3}^{T}}_{40 \times 3} \underbrace{\mathbf{E}}_{3 \times 3} \underbrace{\mathbf{B}_{1}}_{3 \times 40}+\underbrace{\mathbf{B}_{3}^{T}}_{40 \times 3} \underbrace{\mathbf{H}}_{3 \times 3} \underbrace{\mathbf{B}_{3}}_{3 \times 40}+\underbrace{\mathbf{B}_{0 \gamma}^{T}}_{40 \times 2} \underbrace{\bar{A}}_{2 \times 2} \underbrace{\mathbf{B}_{0 \gamma}}_{2 \times 40} \\
& +\underbrace{\mathbf{B}_{0 \gamma}^{T}}_{40 \times 2} \underbrace{\bar{B}}_{2 \times 2} \underbrace{\mathbf{B}_{2 \gamma}}_{2 \times 40}+\underbrace{\mathbf{B}_{2 \gamma}^{T}}_{40 \times 2} \underbrace{\bar{B}}_{2 \times 2} \underbrace{\mathbf{B}_{0 \gamma}}_{2 \times 40}+\underbrace{\mathbf{B}_{2 \gamma}^{T}}_{40 \times 2} \underbrace{\bar{B}}_{2 \times 2} \underbrace{\mathbf{B}_{2 \gamma}}_{2 \times 40}+k_{w} \underbrace{\mathbf{N}_{w}^{T}}_{40 \times 1} \underbrace{\mathbf{N}_{w}}_{1 \times 40}+k_{s}(\underbrace{\left(\frac{\partial \mathbf{N}_{w}}{\partial x}\right)^{T}}_{40 \times 1} \underbrace{\left(\frac{\partial \mathbf{N}_{w}}{\partial x}\right)}_{1 \times 40}+\underbrace{\left(\frac{\partial \mathbf{N}_{w}}{\partial y}\right)^{T}}_{40 \times 1} \underbrace{\left(\frac{\partial \mathbf{N}_{w}}{\partial y}\right)}_{1 \times 40})) \mathrm{d} S,
\end{aligned}
$$

$$
\underbrace{\mathbf{K}_{u \phi e}^{a}}_{40 \times 1}=\int_{S_{e}}(-\underbrace{\mathbf{B}_{0}^{T}}_{40 \times 3} \underbrace{\mathbf{A} \mathbf{e}^{T}}_{3 \times 3} \underbrace{\mathbf{B}_{\phi a}}_{3 \times 1}-\underbrace{\mathbf{B}_{1}^{T}}_{40 \times 3} \underbrace{\mathbf{B e}^{T}}_{3 \times 3} \underbrace{\mathbf{B}_{\phi a}}_{3 \times 1}-\underbrace{\mathbf{B}_{3}^{T}}_{40 \times 3} \underbrace{\mathbf{E e}^{T}}_{3 \times 3} \underbrace{\mathbf{B}_{\phi a}}_{3 \times 1}) \mathrm{d} S,
$$




$$
\begin{aligned}
& \underbrace{\mathbf{K}_{u \phi e}^{s}}_{40 \times 1}=\int_{S_{e}}(-\underbrace{\mathbf{B}_{0}^{T}}_{40 \times 3} \underbrace{\mathbf{A} \mathbf{e}^{T}}_{3 \times 3} \underbrace{\mathbf{B}_{\phi s}}_{1 \times 3}-\underbrace{\mathbf{B}_{1}^{T}}_{40 \times 3} \underbrace{\mathbf{B e}^{T}}_{3 \times 3} \underbrace{\mathbf{B}_{\phi s}}_{1 \times 3}-\underbrace{\mathbf{B}_{3}^{T}}_{40 \times 3} \underbrace{\mathbf{E e}^{T}}_{3 \times 3} \underbrace{\mathbf{B}_{\phi s}}_{1 \times 3}) \mathrm{d} S, \\
& \underbrace{\mathbf{K}_{\phi u e}^{a}}_{1 \times 40}=\int_{S_{e}}(-\underbrace{\mathbf{B}_{\phi a}^{T}}_{1 \times 3} \underbrace{\mathbf{e A}}_{3 \times 3} \underbrace{\mathbf{B}_{0}}_{3 \times 40}-\underbrace{\mathbf{B}_{\phi a}^{T}}_{1 \times 3} \underbrace{\mathbf{e B}}_{3 \times 3} \underbrace{\mathbf{B}_{1}}_{3 \times 40}-\underbrace{\mathbf{B}_{\phi a}^{T}}_{1 \times 3} \underbrace{\mathbf{e E}}_{3 \times 3} \underbrace{\mathbf{B}_{3}}_{3 \times 40}) \mathrm{d} S, \\
& \underbrace{\mathbf{K}_{\phi u e}^{s}}_{1 \times 40}=\int_{S_{e}}(-\underbrace{\mathbf{B}_{\phi s}^{T}}_{1 \times 3} \underbrace{\mathbf{e A}}_{3 \times 3} \underbrace{\mathbf{B}_{0}}_{3 \times 40}-\underbrace{\mathbf{B}_{\phi s}^{T}}_{1 \times 3} \underbrace{\mathbf{e B}}_{3 \times 3} \underbrace{\mathbf{B}_{1}}_{3 \times 40}-\underbrace{\mathbf{B}_{\phi s}^{T}}_{1 \times 3} \underbrace{\mathbf{e}}_{3 \times 3} \underbrace{\mathbf{B}_{3}}_{3 \times 40}) \mathrm{d} S, \\
& \underbrace{\mathbf{K}_{\phi \phi e}^{a}}_{1 \times 1}=\int_{S_{e}} \underbrace{\mathbf{B}_{\phi a}^{T}}_{1 \times 3} \underbrace{\mathbf{p}}_{3 \times 3} \underbrace{\mathbf{B}_{\phi a}}_{3 \times 1} \mathrm{~d} S, \\
& \underbrace{\mathbf{K}_{\phi \phi e}^{s}}_{1 \times 1}=\int_{S_{e}} \underbrace{\mathbf{B}_{\phi s}^{T}}_{1 \times 3} \underbrace{\mathbf{p}}_{3 \times 3} \underbrace{\mathbf{B}_{\phi s}}_{3 \times 1} \mathrm{~d} S, \\
& \underbrace{\widetilde{F}_{e}}_{40 \times 1}=\int_{S_{e}} \underbrace{\mathbf{N}^{T}}_{40 \times 5} \underbrace{\widetilde{F}}_{5 \times 1} \cdot \mathrm{d} S_{e}, \\
& \underbrace{\mathbf{F}_{q e}^{a}}_{1 \times 1}=\int_{S_{e}} \underbrace{\mathbf{B}_{\phi s}^{T}}_{1 \times 3} \underbrace{\{0 ; 0 ; V\}}_{3 \times 1} \mathrm{~d} S ; \\
& \underbrace{\mathbf{F}_{q e}^{s}}_{1 \times 1}=\int_{S_{e}} \underbrace{\mathbf{B}_{\phi s}^{T}}_{1 \times 3} \underbrace{\{0 ; 0 ; V\}}_{3 \times 1} \mathrm{~d} S \text {. }
\end{aligned}
$$

So for the plate element resting on a two-parameter elastic foundation, two components $k_{w} \mathbf{N}_{w}^{T} \mathbf{N}_{w}+k_{s}\left(\left(\partial N_{w} /\right.\right.$ $\left.\partial x)^{T}\left(\partial N_{w} / \partial x\right)+\left(\partial N_{w} / \partial y\right)^{T}\left(\partial N_{w} / \partial y\right)\right)$ will be added to the element stiffness matrix as shown in equation (21). For the plate element with the foundation, these two components are neglected. For the convenience of calculation, the element mesh will be divided so that the element node points will be on the boundary of the elastic domain, which means that an element will lie entirely or not in the domain of the elastic foundation.

For the static problem, minimizing the total potential energy function by variables, we have the following equation:

$$
\left\{\begin{array}{l}
\mathbf{K}_{u u e} \mathbf{q}_{e}+\mathbf{K}_{u \phi e}^{a} \phi_{a e}+\mathbf{K}_{u \phi e}^{s} \phi_{s e}=\mathbf{P}_{e} \\
\mathbf{K}_{\phi u e}^{a} \mathbf{q}_{e}+\mathbf{K}_{\phi \phi e}^{a} \phi_{a e}=\mathbf{F}_{q e}^{a} \\
\mathbf{K}_{\phi u e}^{s} \mathbf{q}_{e}+\mathbf{K}_{\phi \phi e}^{s} \phi_{s e}=\mathbf{F}_{q e}^{s}
\end{array}\right.
$$

Equation (29) can be shortened in the form with only displacement variable $\mathbf{q}_{e}$ as

$$
\left(\mathbf{K}_{u u e}+\mathbf{K}_{u \phi e}^{a} \mathbf{K}_{\phi \phi e}^{a-1} \mathbf{K}_{\phi u e}^{a}+\mathbf{K}_{u \phi e}^{s} \mathbf{K}_{\phi \phi e}^{s-1} \mathbf{K}_{\phi u e}^{s}\right) \mathbf{q}_{e}=\mathbf{P}_{e}+\mathbf{K}_{u \phi e}^{s} \mathbf{F}_{q e}^{s}+\mathbf{K}_{u \phi e}^{a} \mathbf{F}_{q e}^{a} .
$$

Therefore, the static equilibrium equation of the whole structure has the following form:

$$
\begin{aligned}
& \sum_{e}\left(\mathbf{K}_{u u e}+\mathbf{K}_{u \phi e}^{a} \mathbf{K}_{\phi \phi e}^{a-1} \mathbf{K}_{\phi u e}^{a}+\mathbf{K}_{u \phi e}^{s} \mathbf{K}_{\phi \phi e}^{s-1} \mathbf{K}_{\phi u e}^{s}\right) \mathbf{q}_{e} \\
& =\sum_{e}\left(\mathbf{P}_{e}+\mathbf{K}_{u \phi e}^{s} \mathbf{F}_{q e}^{s}+\mathbf{K}_{u \phi e}^{a} \mathbf{F}_{q e}^{a}\right) .
\end{aligned}
$$

The kinetic energy of the plate element is determined by formula

$$
T_{e}=\frac{1}{2} \int_{V_{e}} \dot{u}^{T} \rho(x, z) \dot{u} \mathrm{~d} S \mathrm{~d} z=\frac{1}{2} \dot{q}_{e}^{T}\left(\begin{array}{c}
\int_{V_{e}} \mathbf{N}^{T} \mathbf{L}^{T} \rho_{p}(x) \mathbf{L} \mathbf{N} \mathrm{d} S \mathrm{~d} z+\int_{V_{e}} \mathbf{N}^{T} \mathbf{L}^{T} \rho_{a}(x) \mathbf{L N} \mathrm{d} S \mathrm{~d} z \\
\int_{V_{e}} \mathbf{N}^{T} \mathbf{L}^{T} \rho_{s}(x) \mathbf{L N} \mathrm{N} S \mathrm{~d} z
\end{array}\right) \dot{q}_{e}=\frac{1}{2} \dot{q}_{e}^{T} \mathbf{M}_{e} \dot{q}_{e},
$$


in which $\rho_{p}, \rho_{a}$, and $\rho_{s}$ are the densities of the plate, actuator layer, and sensor layer, respectively; $\mathbf{N}$ is the shape function matrix; $\mathbf{L}$ and the element mass matrix $\mathbf{M}_{e}$ are calculated as follows:

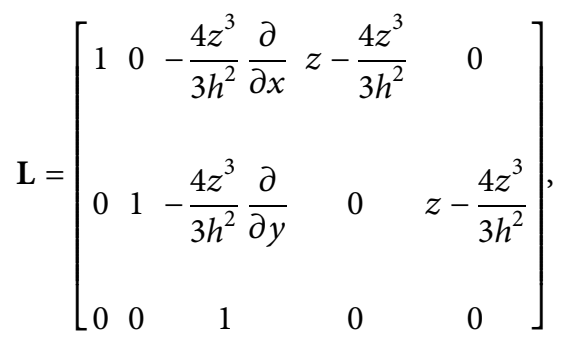

$$
\begin{aligned}
& \mathbf{M}_{e}=\left(\int_{V_{e}} \mathbf{N}^{T} \mathbf{L}^{T} \rho_{p}(x) \mathbf{L} \mathbf{N d} S \mathrm{~d} z+\int_{V_{e}} \mathbf{N}^{T} \mathbf{L}^{T} \rho_{a}(x) \mathbf{L} \mathbf{N d} S \mathrm{~d} z\right. \\
& \left.+\int_{V_{e}} \mathbf{N}^{T} \mathbf{L}^{T} \rho_{s}(x) \mathbf{L N d} S \mathrm{~d} z\right) .
\end{aligned}
$$

For the free vibration problem, to find frequencies of the plate, the following equation is solved:

$$
\left(\sum_{e}\left\{\mathbf{K}_{u u e} \mathbf{q}_{e}+\mathbf{K}_{u \phi e}^{a} \phi_{a e}+\mathbf{K}_{u \phi e}^{s} \phi_{s e}\right\}-\omega^{2} \sum_{e} \mathbf{M}_{e}\right) \mathbf{q}_{e}=0 .
$$

Hence, the voltage applied to the structure $V$ will generate an electric force vector as shown in equation (29), thereby affecting the static displacement of the structure. This paper has not considered the effect of this potential on the vibration response of the structure.

Through the above calculation formulas, using Reddy's higher-order shear deformation theory, the element stiffness matrix and the element mass matrix show more components, leading to more complicated calculations than the classical plate theory and the FSDT, and no shear correction factor is required. By using the 8-node plate element and the finite element method, the equilibrium equation of the structure is derived. To verify the reliability of the proposed theory and mathematical model, the following section will present a specific comparison to determine this problem.

\section{Numerical Results and Discussion}

\subsection{Free Vibration for Piezoelectric FGM Plate}

4.1.1. Accuracy Study. Consider that a piezoelectric FGM (Ti-6Al-4V/aluminum oxide) plate with the dimensions $a=b=0.4 \mathrm{~m}$, the thickness $h=5 \mathrm{~mm}$, the thicknesses of two piezoelectric layers $h_{a}=h_{s}=0.1 \mathrm{~mm}$, two piezoelectric layers $\mathrm{G}-1195 \mathrm{~N}$ with $E_{p i}=63 \mathrm{GPa}$, Poison's ratio 0.3, $d_{31}=d_{32}=254.10^{-12} \mathrm{~m} / \mathrm{V}$, and $k_{33}=15 \mathrm{nF} / \mathrm{m}$ is considered. The plate is fully and simply supported (SSSS), and the first natural frequencies of the plate obtained from this numerical work, FEM and Kirchoff-love plate theory [51], and the analytical solution [56] are presented in Table 1. Herein,
Table 1 also presents the numerical results with different meshes. It can be seen clearly that an $8 \times 8$ mesh can return accurate data. For this mesh, in Table 2, pre-Kirchoff-love plate sends the first ten natural frequencies of the piezoelectric FGM plate. From Tables 1 and 2, the proposed method is verified. For all the following investigations, an $8 \times 8$ mesh is used.

Next, the effect of the elastic foundation (EF) is considered; the plate is placed on the elastic foundation with two parameters. The FGM $\left(\mathrm{Al} / \mathrm{ZrO}_{2}\right)$ plate has $a=0.2 \mathrm{~m}$ and $b=a / 1.5$, the plate thickness of $h=a / 10$ and $h=a / 20$, and the material properties $E_{c}=200 \mathrm{GPa}, \nu_{c}=0.3, \rho_{c}=5700 \mathrm{~kg} / \mathrm{m}^{3}$, $E_{m}=70 \mathrm{GPa}, v_{m}=0.3$, and $\rho_{m}=2702 \mathrm{~kg} / \mathrm{m}^{3}$. The plate is fully and simply supported. Two nondimensional parameters of the $\mathrm{EF}$ are given as follows:

$$
\left\{\begin{array}{l}
K_{w}^{*}=\frac{K_{w} a^{4}}{A}, \\
K_{s}^{*}=\frac{K_{s} a^{2}}{A},
\end{array}\right.
$$

with

$$
A=\frac{h^{3}}{\left(1-v_{m}^{2}\right)}\left[\frac{n\left(8+3 n+n^{2}\right) E_{m}+3\left(2+n+n^{2}\right) E_{c}}{12(1+n)(2+n)(3+n)}\right] .
$$

The nondimensional natural frequency of the plate is defined as

$$
\omega^{*}=\omega h \sqrt{\frac{\rho_{c}}{E_{c}}} .
$$

Table 3 presents the nondimensional natural frequencies of the plate with different thicknesses and volume fraction indexes obtained from this work and the analytical solution of Hosseini-Hashemi et al. [57]. It can be easily seen that the results have a good agreement.

4.1.2. Numerical Results Study for Free Vibration of Piezoelectric FGM Plates. This section investigates the effect of two parameters of the EF on natural frequencies of the piezoelectric FGM plate $\left(\mathrm{Al} / \mathrm{ZrO}_{2}\right)$ with $a=b=0.2 \mathrm{~m}$, the thickness of $h=a /$ 20 and $h_{a}=h_{s}=h / 20$, and the material properties $E_{c}=200 \mathrm{GPa}$, $v_{c}=0.3, \quad \rho_{c}=5700 \mathrm{~kg} / \mathrm{m}^{3}, \quad E_{m}=70 \mathrm{GPa}, \quad v_{m}=0.3, \quad$ and $\rho_{m}=2702 \mathrm{~kg} / \mathrm{m}^{3}$; two piezoelectric layers are made of G-1195N with the material properties as shown in Section 4.1.1. The nondimensional frequency and two parameters of the EF are calculated as equations (36) and (38), respectively. For boundary conditions, some descriptions are as follows. If one edge of the plate is simply supported, it will be denoted as $S$. Similarly, $C$ and $F$ denote, respectively, clamp support and free support. Therefore, the plate is fully and simply supported, called SSSS, the plate is clamped at one edge, and the other three edges are free, called $C F F F$.

(1) Effect of the Stiffness of the Elastic Foundation. Consider an elastic foundation with two parameters, $K_{s}^{*}$ varies from 0 
TABLE 1: The comparison of first natural frequencies $(\mathrm{Hz})$ with different methods and meshes.

\begin{tabular}{|c|c|c|c|c|c|c|c|c|c|}
\hline \multirow{2}{*}{ Mode } & \multirow{2}{*}{ Method } & \multirow{2}{*}{ Mesh } & \multicolumn{7}{|c|}{$n$} \\
\hline & & & 0 & 0.2 & 0.5 & 1 & 5 & 15 & 1000 \\
\hline \multirow{7}{*}{1} & Authors [51] & - & 144.2 & 168.7 & 185.4 & 198.9 & 230.4 & 247.3 & 261.7 \\
\hline & Authors [56] & - & 145.3 & 169.2 & 186.2 & 200.5 & 233.0 & 250.3 & 265.1 \\
\hline & & $4 \times 4$ & 146.1 & 170.6 & 175.5 & 201.7 & 233.8 & 250.7 & 265.1 \\
\hline & & $6 \times 6$ & 145.4 & 169.8 & 186.6 & 200.7 & 232.5 & 249.4 & 263.8 \\
\hline & Present FE solution & $8 \times 8$ & 145.4 & 169.8 & 186.6 & 200.6 & 232.4 & 249.3 & 263.7 \\
\hline & & $10 \times 10$ & 145.4 & 169.8 & 186.6 & 200.6 & 232.4 & 249.3 & 263.7 \\
\hline & & $12 \times 12$ & 145.4 & 169.8 & 186.6 & 200.6 & 232.4 & 249.3 & 263.7 \\
\hline
\end{tabular}

TABLE 2: The first ten natural frequencies of the piezoelectric FGM plate $(8 \times 8$ mesh).

\begin{tabular}{|c|c|c|c|c|c|c|c|c|}
\hline \multirow{2}{*}{ Mode } & \multirow{2}{*}{ Method } & \multicolumn{7}{|c|}{$n$} \\
\hline & & 0 & 0.2 & 0.5 & 1 & 5 & 15 & 1000 \\
\hline \multirow{3}{*}{1} & Authors [51] & 144.2 & 168.7 & 185.4 & 198.9 & 230.4 & 247.3 & 261.7 \\
\hline & Authors [56] & 145.3 & 169.2 & 186.2 & 200.5 & 233.0 & 250.3 & 265.1 \\
\hline & Present FE solution & 145.4 & 169.8 & 186.6 & 200.6 & 232.4 & 249.3 & 263.7 \\
\hline \multirow{3}{*}{2} & Authors [51] & 359.0 & 420.6 & 462.4 & 495.6 & 573.8 & 615.5 & 651.4 \\
\hline & Authors [56] & 363.0 & 422.6 & 465.2 & 500.9 & 582.1 & 625.2 & 662.2 \\
\hline & Present FE solution & 363.3 & 424.3 & 466.3 & 501.5 & 581.0 & 623.1 & 659.1 \\
\hline \multirow{3}{*}{3} & Authors [51] & 359.0 & 420.6 & 462.4 & 495.6 & 573.8 & 615.5 & 651.4 \\
\hline & Authors [56] & 363.0 & 422.6 & 465.2 & 500.9 & 582.1 & 625.2 & 662.2 \\
\hline & Present FE solution & 363.3 & 424.3 & 466.3 & 501.5 & 581.0 & 623.1 & 659.1 \\
\hline \multirow{3}{*}{4} & Authors [51] & 564.1 & 665.0 & 731.1 & 778.9 & 902.0 & 967.7 & 1024.2 \\
\hline & Authors [56] & 580.3 & 675.6 & 743.6 & 800.9 & 930.6 & 999.6 & 1058.5 \\
\hline & Present FE solution & 581.4 & 679.0 & 746.3 & 802.6 & 930.0 & 997.4 & 1054.9 \\
\hline \multirow{3}{*}{5} & Authors [51] & 717.8 & 841.2 & 925.4 & 993.1 & 1148.1 & 1231.0 & 1302.6 \\
\hline & Authors [56] & 725.0 & 844.0 & 929.0 & 1000.5 & 1162.6 & 1248.8 & 1322.4 \\
\hline & Present FE solution & 727.6 & 849.8 & 933.9 & 1004.4 & 1163.8 & 1248.2 & 1320.2 \\
\hline \multirow{3}{*}{6} & Authors [51] & 717.8 & 841.2 & 925.4 & 993.1 & 1148.1 & 1231.0 & 1302.6 \\
\hline & Authors [56] & 725.0 & 844.0 & 929.0 & 1000.5 & 1162.6 & 1248.8 & 1322.4 \\
\hline & Present FE solution & 727.6 & 849.8 & 933.9 & 1004.4 & 1163.8 & 1248.2 & 1320.2 \\
\hline \multirow{3}{*}{7} & Authors [51] & 908.2 & 1073.7 & 1180.9 & 1255.9 & 1453.3 & 1558.7 & 1649.7 \\
\hline & Authors [56] & 941.6 & 1096.2 & 1206.7 & 1299.6 & 1510.1 & 1622.1 & 1717.6 \\
\hline & Present FE solution & 947.4 & 1106.6 & 1216.3 & 1308.5 & 1516.5 & 1626.2 & 1719.6 \\
\hline \multirow{3}{*}{8} & Authors [51] & 908.2 & 1073.7 & 1180.9 & 1255.9 & 1453.3 & 1558.7 & 1649.7 \\
\hline & Authors [56] & 941.6 & 1096.2 & 1206.7 & 1299.6 & 1510.1 & 1622.1 & 1717.6 \\
\hline & Present FE solution & 947.4 & 1106.6 & 1216.3 & 1308.5 & 1516.5 & 1626.2 & 1719.6 \\
\hline \multirow{3}{*}{9} & Authors [51] & 1223.1 & 1432.1 & 1576.9 & 1697.1 & 1958.1 & 2097.9 & 2219.6 \\
\hline & Authors [56] & 1229.8 & 1431.7 & 1576.2 & 1697.6 & 1972.7 & 2118.8 & 2243.6 \\
\hline & Present FE solution & 1243.5 & 1452.4 & 1596.2 & 1716.9 & 1989.5 & 2133.7 & 2256.6 \\
\hline \multirow{3}{*}{10} & Authors [51] & 1223.1 & 1432.1 & 1576.9 & 1697.1 & 1958.1 & 2097.9 & 2219.6 \\
\hline & Authors [56] & 1229.8 & 1431.7 & 1576.2 & 1697.6 & 1972.7 & 2118.8 & 2243.6 \\
\hline & Present FE solution & 1243.5 & 1452.4 & 1596.2 & 1716.9 & 1989.5 & 2133.7 & 2256.6 \\
\hline
\end{tabular}

to 500 , and $K_{w}^{*}$ changes in the range from 0 to 10000 , and the volume fraction index $n=1$. The plate is rested on one area of the elastic foundation with $d_{e}=a / 3$. The boundary condition is SSSS. The first five natural frequencies are shown in Figure 2. From these results, it can be seen that when increasing the values of two elastic foundation parameters, natural frequencies increase. Particularly, when $K_{s}^{*}$ gets the small value, the curve of the natural frequency tends to depend on $K_{w}^{*}$ much.

(2) Effect of the Length of the Elastic Foundation Area $d_{e}$. In this investigation, $K_{w}^{*}$ varies from 0 to 10000 while $K_{s}^{*}=50$, the volume fraction index $n=1$, and the plate is fully and simply supported (SSSS). The plate is rested on one area of the elastic foundation with $d_{e}$ varying from $a / 12$ to $a / 2$. The nondimensional natural frequencies of the plate are presented in Figure 3. One can see that when increasing $d_{e}$, which means increasing the area of the elastic foundation, the plate becomes stronger; therefore, the natural frequency of the plate increases. At the same time, when increasing the stiffness parameter $K_{w}^{*}$, the plate also becomes harder, and the first natural frequency of the plate increases.

Five natural vibration mode shapes of the plate in cases of $d_{e}=a / 12$ and $d_{e}=a / 2$ are shown in Figure 4 . It can be seen 
TABle 3: The nondimensional natural frequency $\omega$ of fully and simply supported FGM plate resting on the two-parameter foundation (SSSS).

\begin{tabular}{lcccc}
\hline$\left(K_{w}^{*}, K_{s}^{*}\right)$ & $a / h$ & $n$ & Authors [57] & Present FE solution \\
\hline & & 0 & 0.0239 & 0.0239 \\
& 0.25 & 0.0226 & 0.0231 \\
& 20 & 1 & 0.0215 & 0.0220 \\
$(0,0)$ & & 5 & 0.0218 & 0.0225 \\
& & 0 & 0.0204 & 0.0206 \\
& & 0.25 & 0.0918 & 0.0919 \\
& 10 & 1 & 0.0860 & 0.0888 \\
& & 5 & 0.0815 & 0.0848 \\
& & $\infty$ & 0.0789 & 0.0860 \\
& & 0.25 & 0.0342 & 0.0790 \\
\hline \multirow{4}{*}{20} & 1 & 0.0328 & 0.0342 \\
& & 5 & 0.0318 & 0.0331 \\
& & $\infty$ & 0.0293 & 0.0321 \\
& & 0 & 0.1336 & 0.0328 \\
& & 0.25 & 0.1277 & 0.0294 \\
& 10 & 1 & 0.1238 & 0.1337 \\
& & 5 & 0.1253 & 0.1295 \\
& & $\infty$ & 0.1148 & 0.1258 \\
& & &
\end{tabular}

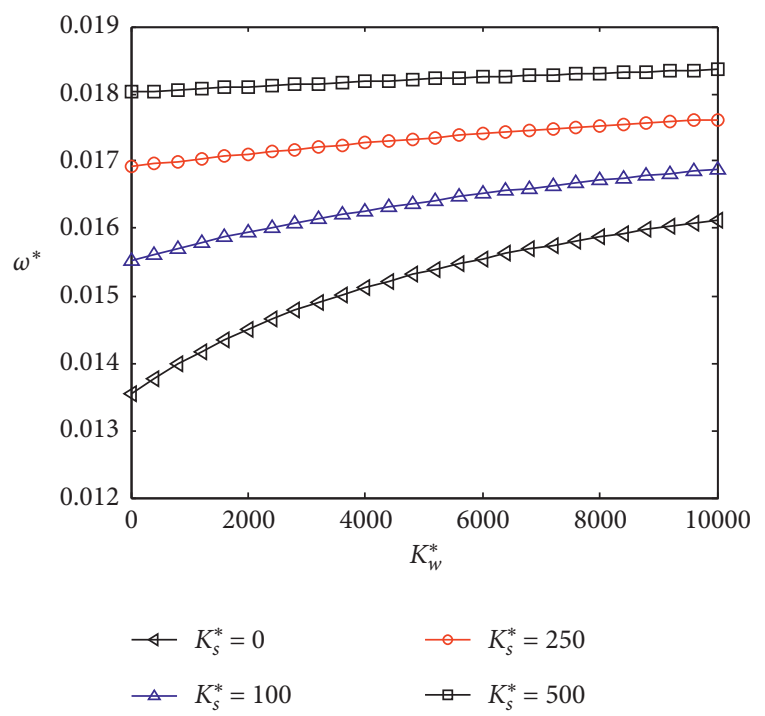

Figure 2: Effect of $K_{w}^{*}$ and $K_{s}^{*}$ on nondimensional natural frequencies $\omega^{*}$ of the plate.

clearly that the higher the length $d_{\mathrm{e}}$ is, the stronger the nondimensional natural frequencies as well as vibration mode shapes are affected, especially for the first natural frequency of the structure. Herein, to obtain the higher quality of vibration mode shapes, $12 \times 12$ mesh is used instead of $8 \times 8$ mesh as mentioned.

(3) Effect of Volume Fraction Index $n$. The plate is rested on one area of the elastic foundation with the length $d_{e}=a / 3$. In this exploration, when $K_{s}^{*}=50$, four cases of $K_{w}^{*}$ are considered, which are $0,100,500$, and 1000 , and in the case of

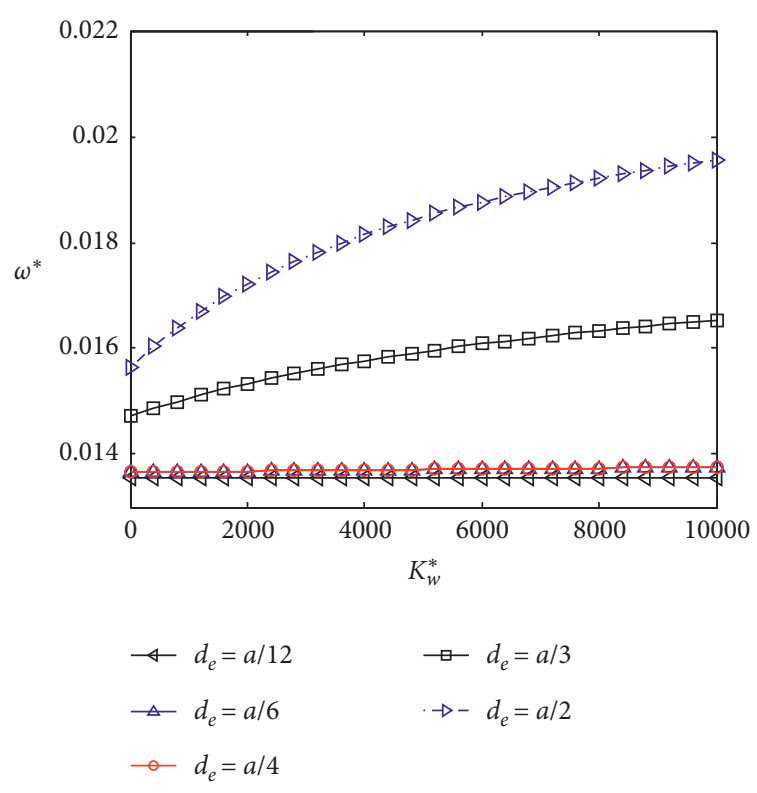

Figure 3: Effect of $K_{w}^{*}$ and $d_{e}$ on nondimensional natural frequencies $\omega^{*}$ of the plate, $K_{s}^{*}=50$.

$K_{w}^{*}=1000, K_{s}^{*}$ gets values in a range of $0,10,50$, and 100 . The plate is SSSS. Figures 5 and 6 present the nondimensional natural frequencies of the plate changes as a function of the volume fraction index $n$ from 0 to 10 . At the same time, the value of the volume fraction index in a range of 0.6 to 1.8 is found so that the first natural frequency of the plate is the smallest.

(4) Effect of Boundary Condition. In this section, the effect of boundary condition on the nondimensional natural frequencies of the plate is investigated. Four cases of boundary condition are considered: SSSS, CCCC, CFFF, and SCSC. Figure 7 presents the dependence of the nondimensional natural frequencies on the volume fraction index $n$ and boundary condition. It can be observed that when $n$ increases, the nondimensional natural frequencies go down sharply and then go up gradually. They reach the minimum values around $n=1.5$. Figure 8 shows the dependence of the nondimensional natural frequencies on $K_{w}^{*}$ with four boundary conditions $\left(K_{s}^{*}=50, n=1\right)$. It can be seen that when $K_{w}^{*}$ increases, $\omega^{*}$ does not change much.

\subsection{Static Bending of Piezoelectric FGM Plate}

4.2.1. Verification Problem. Firstly, the deflection of a piezoelectric FGM plate $\left(\mathrm{Al}_{2} \mathrm{O}_{3} / \mathrm{Ti}-6 \mathrm{Al}-4 \mathrm{~V}\right)$ with dimensions $a=b=0.4 \mathrm{~m}, h=5 \mathrm{~mm}$, and $h_{a}=h_{s}=0.1 \mathrm{~mm}$ are considered; material properties of the core layer are as follows: $E_{c}=320.24 \mathrm{GPa}$, Poisson's ratio of ceramic $0.26, E_{m}=105.7$ $\mathrm{GPa}$, and Poisson's ratio of metal 0.2981 . The face sheet layers are made from the piezoelectric material G-1195N, where material properties are given in Section 4.1.1. The plate is $C F F F$ and subjected to uniformly distributed load $100 \mathrm{~N} / \mathrm{m}^{2}$ and actuator voltage $V=0$ and $40 \mathrm{~V}$. The 


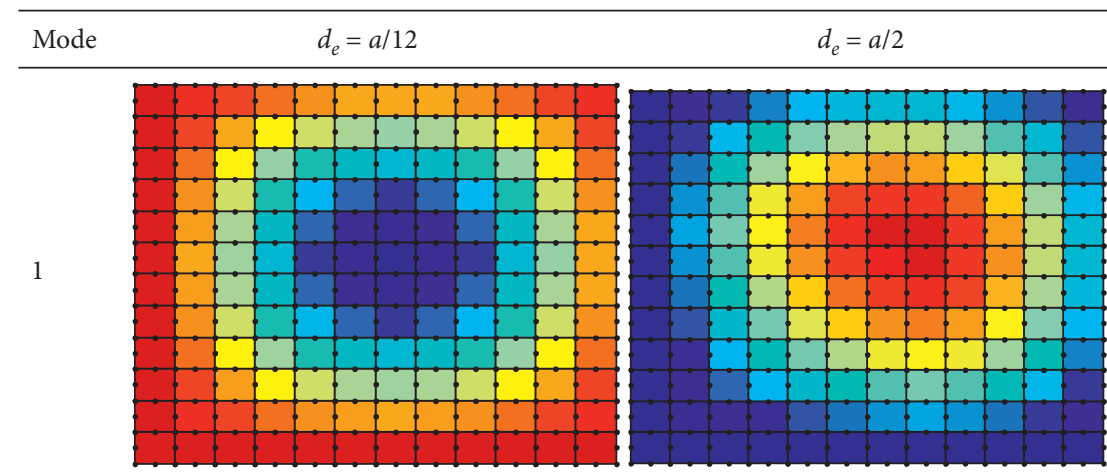

2

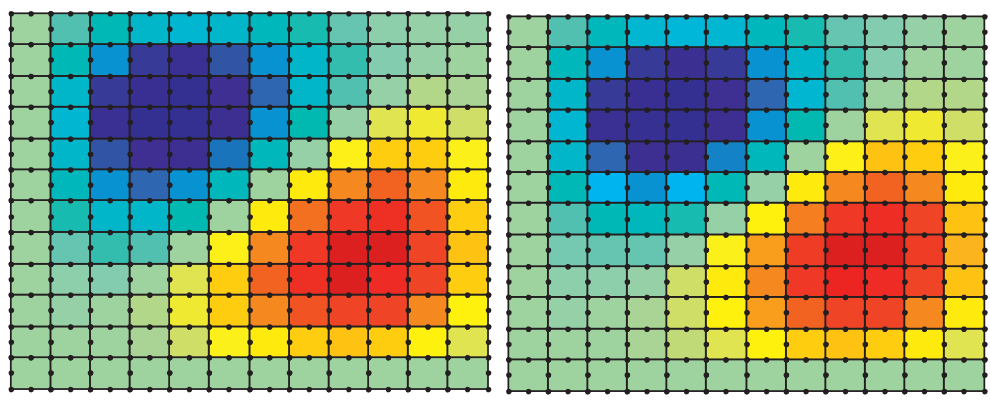

3

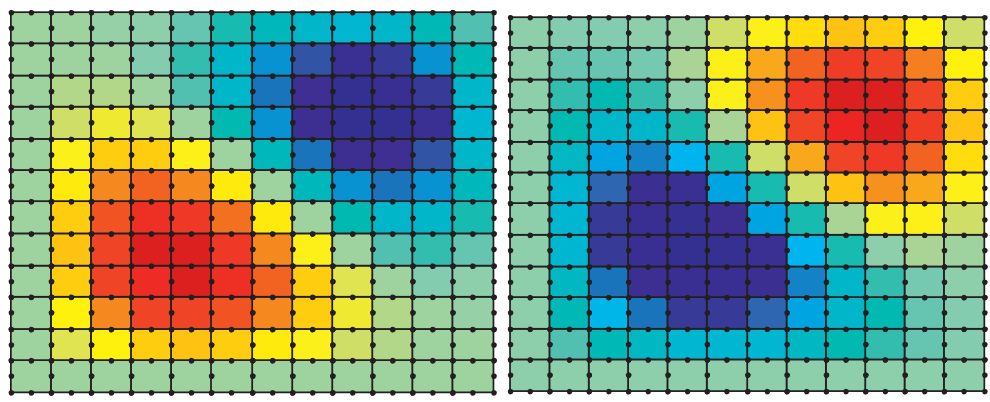

4
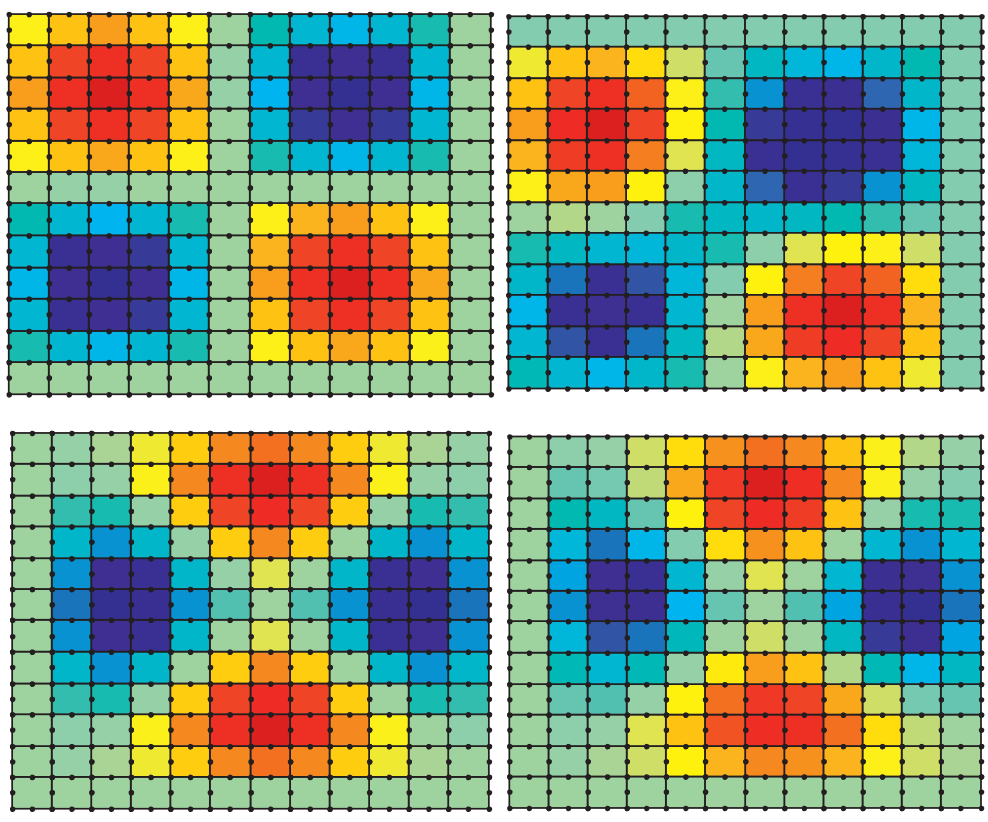

Figure 4: First five natural vibration mode shapes, $K_{w}^{*}=500$ and $K_{s}^{*}=50, n=1$. 


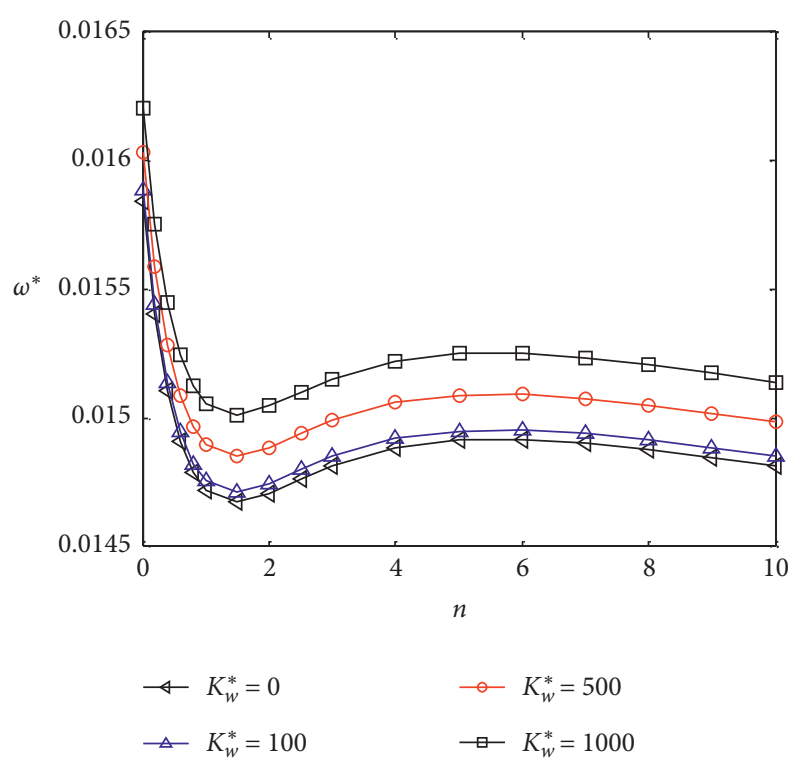

FIGURE 5: Effect of $n$ on nondimensional frequencies of piezoelectric FGM plate with the values of $K_{w}^{*}$ and $K_{s}^{*}=50$.

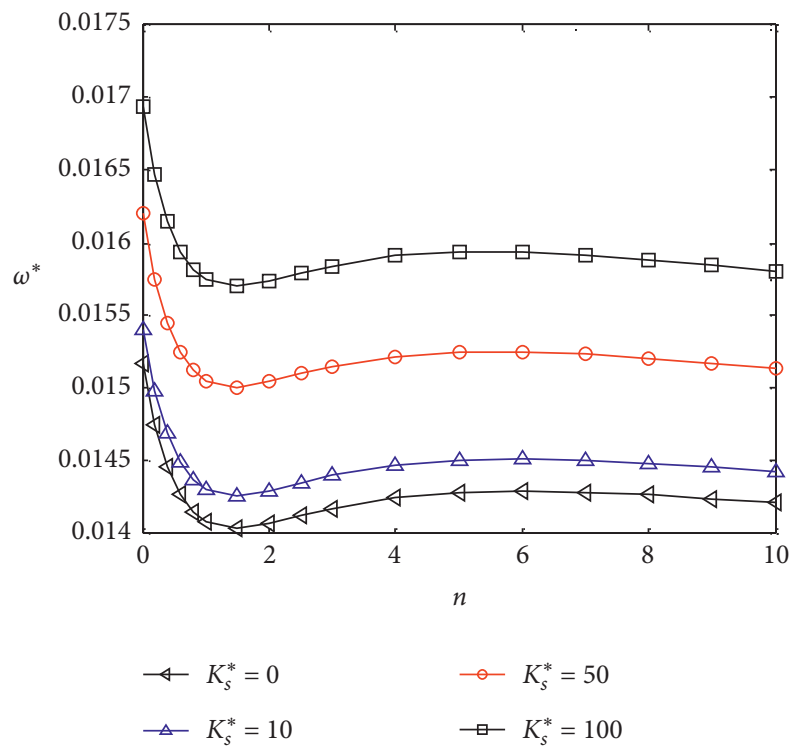

FIgURE 6: Effect of $n$ on nondimensional frequencies of piezoelectric FGM plate with the values of $K_{s}^{*}, K_{w}^{*}=1000$.

deflections at $y=b / 2$ obtained from this work and [51] are presented in Figures 9 and 10. Note that the upper layer of the plate is metal Ti- $6 \mathrm{Al}-4 \mathrm{~V}$, and the lower one of the plate is ceramic $\mathrm{Al}_{2} \mathrm{O}_{3}$. From these comparisons, it can be concluded that the proposed method is verified.

Next, the plate resting on an elastic foundation is considered. Consider a square plate with $a=b=0.2 \mathrm{~m}, h=a / 10$, and $a /$ 200, the material properties $E_{c}=E_{m}=320.24 \mathrm{GPa}$, and Poisson's ratio 0.26 . The plate is SSSS and under a uniformly distributed load $q_{0}$. The nondimensional elastic foundation parameters are calculated as

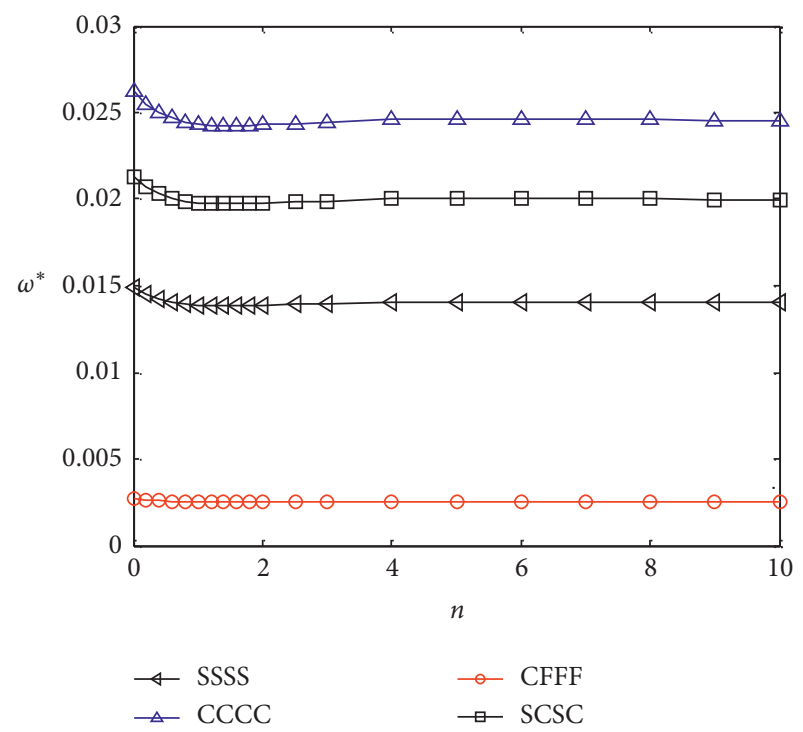

Figure 7: The effect of boundary condition and the volume fraction index $\mathrm{n}$ on nondimensional natural frequencies of the plate, $K_{s}^{*}=10, K_{w}^{*}=100$, and $d_{e}=a / 3$.

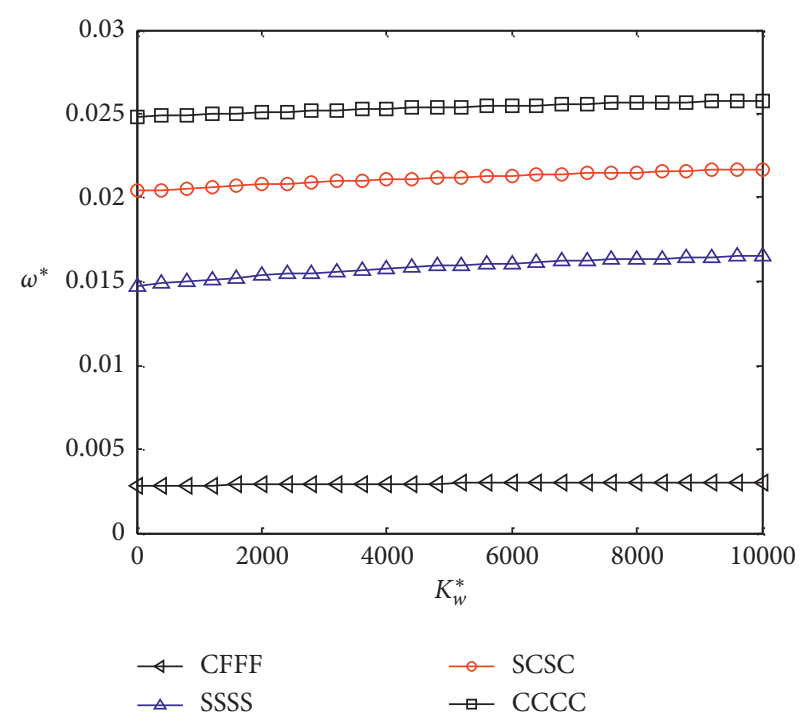

FIgURE 8: The effect of boundary condition and $K_{w}^{*}$ on the nondimensional natural frequencies of the plate, $K_{s}^{*}=50$ and $d_{e}=a / 3$.

$$
\left\{\begin{array}{l}
K_{w}^{*}=\frac{K_{w} a^{4}}{D}, \\
K_{s}^{*}=\frac{K_{s} a^{2}}{D},
\end{array}\right.
$$

with

$$
D=\frac{E_{c} h^{3}}{12\left(1-v_{m}^{2}\right)}
$$




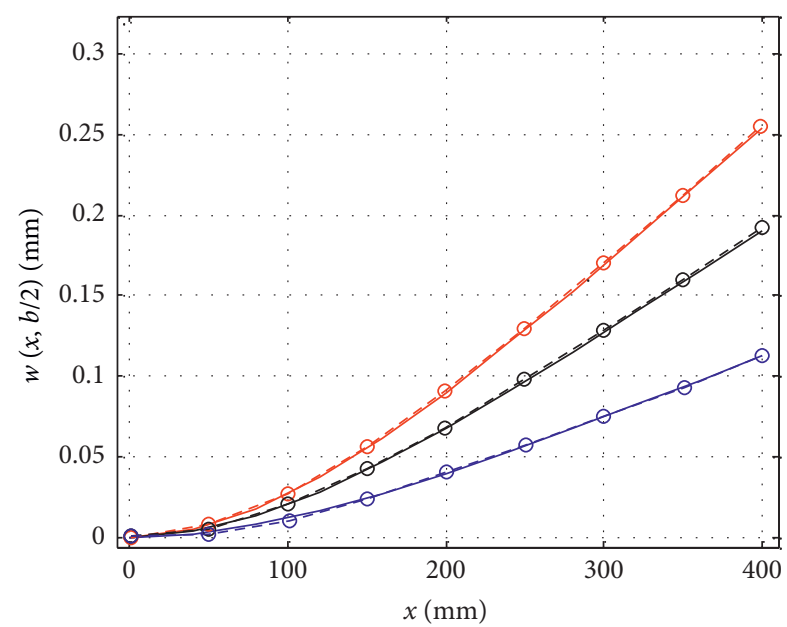

$n=0$ : present FE solution

$n=0.2$ : present FE solution

$-n=5$ : present FE solution

$-\ominus-n=0$ : authors [51]

$-\ominus-n=0.2$ : authors [51]

$-\ominus-n=5$ : authors [51]

FIgURE 9: Deflection $w(x, y=b / 2)$ of the FGM plate under a uniformly distributed load of $100 \mathrm{~N} / \mathrm{m}^{2}$ and actuator voltage $V=0 \mathrm{~V}$.

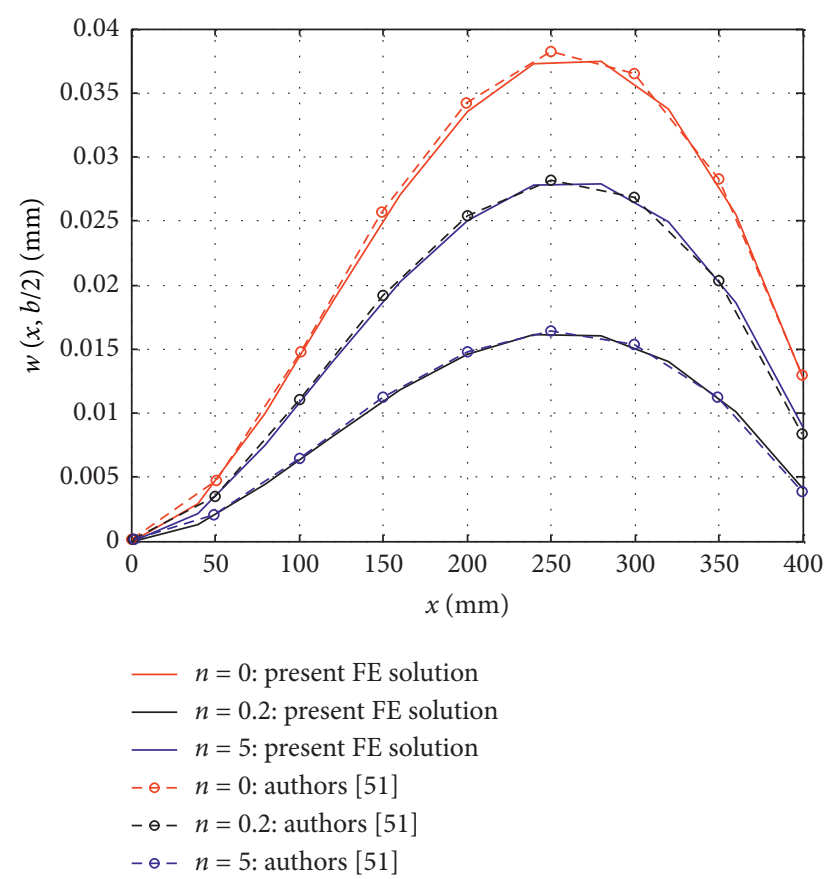

Figure 10: Deflection $w(x, y=b / 2)$ of the FGM plate under a uniformly distributed load of $100 \mathrm{~N} / \mathrm{m}^{2}$ and actuator voltage $V=40 \mathrm{~V}$.

The nondimensional deflection at the center point of the plate is defined by the following formula:

$$
\bar{w}=\frac{10^{3} D}{q_{0} a^{4}} w\left(\frac{a}{2}, \frac{b}{2}\right) .
$$

The comparative results obtained by this work, the differential quadrature method [58], and the analytical method [28] are presented in Table 4. From here, it can conclude that the proposed method is verified.

4.2.2. Numerical Study for Static Bending of Piezoelectric FGM Plate. Consider a piezoelectric FGM plate $\left(\mathrm{Al} / \mathrm{ZrO}_{2}\right)$ with dimensions $a=b=0.2 \mathrm{~m}$, the thickness of $h=a / 20$ and $h_{a}=h_{s}=h / 20$, and the material properties $E_{c}=200 \mathrm{GPa}$, $v_{c}=0.3, E_{m}=70 \mathrm{GPa}$, and $v_{m}=0.3$; the plate has two piezoelectric layers G-1195N with the material properties as shown in Section 4.1.1. The elastic foundation parameters are calculated as shown in equation (39), and the plate is under a uniformly distributed load $q_{0}=10^{5} \mathrm{~N} / \mathrm{m}^{2}$. The maximum nondimensional deflection is standardization as follows:

$$
w^{*}=\frac{10^{3} \mathrm{D} \cdot w_{\min }}{q_{0} a^{4}}
$$

(1) Effect of the Stiffness of the Foundation. Herein, let $K_{s}^{*}$ increase gradually from 0 to 500 while $K_{w}^{*}$ changes in the range from 0 to 10000 . The volume fraction index $n=1$. The plate is rested on one area of the elastic foundation with $d_{e}=a / 3$. The nondimensional defections $w^{*}$ are shown in Figures 11 and 12. These figures indicate that when increasing the stiffness of the elastic foundation, the maximum deflections of the plate decrease; that is due to the increased total energy of the plate due to the addition of the stiffness component of the foundation, so the global stiffness matrix of the structure increases, reducing the deflection of the plate. Particularly, the smaller the value $K_{s}^{*}$ gets, the higher the slope of deflection curve $w^{*}$ depending on $K_{s}^{*}$ is. Besides, when the plate is subjected to both mechanical and electric loads, the maximum deflection of the plate decreases. The reason is that the piezoelectric effect reduces the maximum deflection. This can be explained as follows: when the plate is subjected to both mechanical and electrical loads, the force direction of the electrical loads is in the opposite direction of the mechanical load; therefore, the total force acting on the plate will be reduced, and the deflection of the plate decreases.

(2) Effect of Volume Fraction Index $n$. Parameters of the elastic foundation are $K_{s}^{*}=50, K_{w}^{*}=0-1000$, and $d_{e}=a / 3$. By changing the volume fraction index $n$ in a range of 0 to 10 , the deflection curve $w^{*}$ depending on $n$ in the cases of $V=0$ and $V=40 \mathrm{~V}$ is shown in Figures 13 and 14 . From these figures, it can be seen that when the volume fraction index $n$ increases, the maximum deflection $w^{*}$ increases; this can explain that when the volume fraction index $n$ increases, the portion of the metal increases, the plate becomes softer, and the deflection of the plate $w^{*}$ increases. However, when the 
TABLe 4: Nondimensional deflection $\bar{w}$ of the plate resting on the two-parameter elastic foundation.

\begin{tabular}{|c|c|c|c|c|c|c|c|}
\hline \multirow{2}{*}{$K_{w}^{*}$} & \multirow{2}{*}{$K_{s}^{*}$} & \multicolumn{3}{|c|}{$a / h=10$} & \multicolumn{3}{|c|}{$a / h=200$} \\
\hline & & Authors [58] & Authors [28] & Present FE solution & Authors [58] & Authors [28] & Present FE solution \\
\hline \multirow{4}{*}{1} & 5 & 3.3455 & 3.3455 & 3.3401 & 3.2200 & 3.2200 & 3.2196 \\
\hline & $` 10$ & 2.7505 & 2.7504 & 2.7509 & 2.6684 & 2.6684 & 2.6721 \\
\hline & 15 & 2.3331 & 2.3331 & 2.3364 & 2.2763 & 2.2763 & 2.2818 \\
\hline & 20 & 2.0244 & 2.0244 & 2.0291 & 1.9834 & 1.9834 & 1.9898 \\
\hline \multirow{4}{*}{81} & 5 & 2.8422 & 2.8421 & 2.8448 & 2.7552 & 2.7552 & 2.7610 \\
\hline & $` 10$ & 2.3983 & 2.3983 & 2.4034 & 2.3390 & 2.3390 & 2.3462 \\
\hline & 15 & 2.0730 & 2.0730 & 2.0792 & 2.0306 & 2.0306 & 2.0384 \\
\hline & 20 & 1.8245 & 1.8244 & 1.8311 & 1.7932 & 1.7932 & 1.8011 \\
\hline \multirow{4}{*}{625} & 5 & 1.3785 & 1.3785 & 1.3913 & 1.3688 & 1.3688 & 1.3820 \\
\hline & $' 10$ & 1.2615 & 1.2615 & 1.2732 & 1.2543 & 1.2543 & 1.2663 \\
\hline & 15 & 1.1627 & 1.1627 & 1.1734 & 1.1572 & 1.1572 & 1.1683 \\
\hline & 20 & 1.0782 & 1.0782 & 1.0880 & 1.0740 & 1.0740 & 1.0842 \\
\hline
\end{tabular}

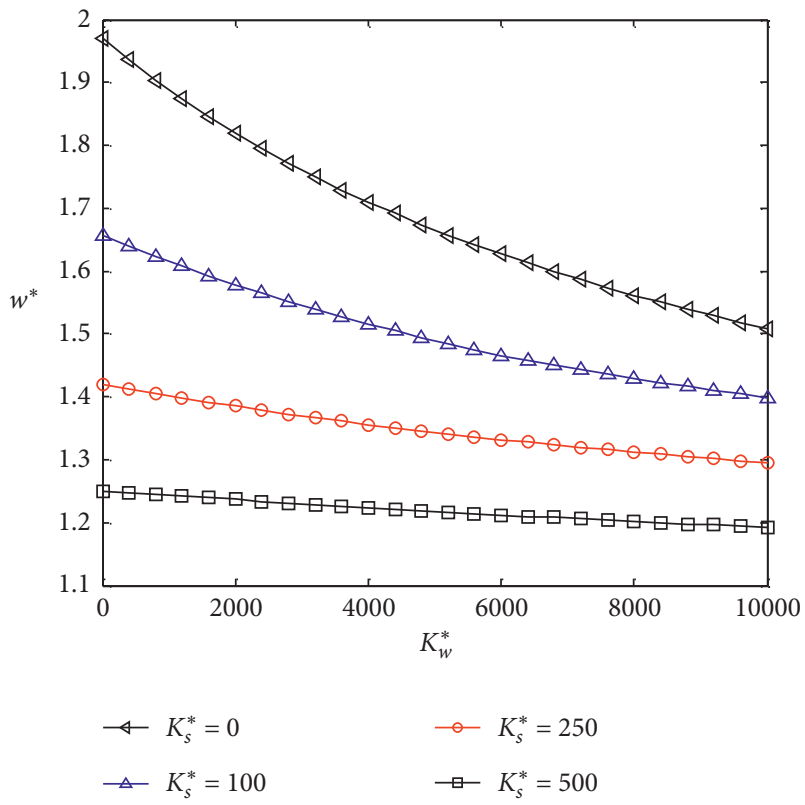

Figure 11: Deflection $w^{*}$ of the FGM plate under a uniformly distributed load of $10^{5} \mathrm{~N} / \mathrm{m}^{2}$ and actuator voltage $V=0 \mathrm{~V}, n=1$, $K_{s}^{*}=0-500$ and $K_{w}^{*}=0-10000$.

piezoelectric effect is applied to the plate, the maximum deflection $w^{*}$ decreases.

(3) Effect of Boundary Condition and the Area of the Elastic Foundation. In this subsection, the plate is rested on the elastic foundation with $K_{s}^{*}=10$ and $K_{w}^{*}=100$. Three cases of boundary condition SSSS, CSCS, and SSCC are considered. The volume fraction index $n=1$. By increasing gradually the length of the area of the elastic foundation $d_{e}$ in a range of 0 to $a / 2$, nondimensional deflections $w^{*}$ with

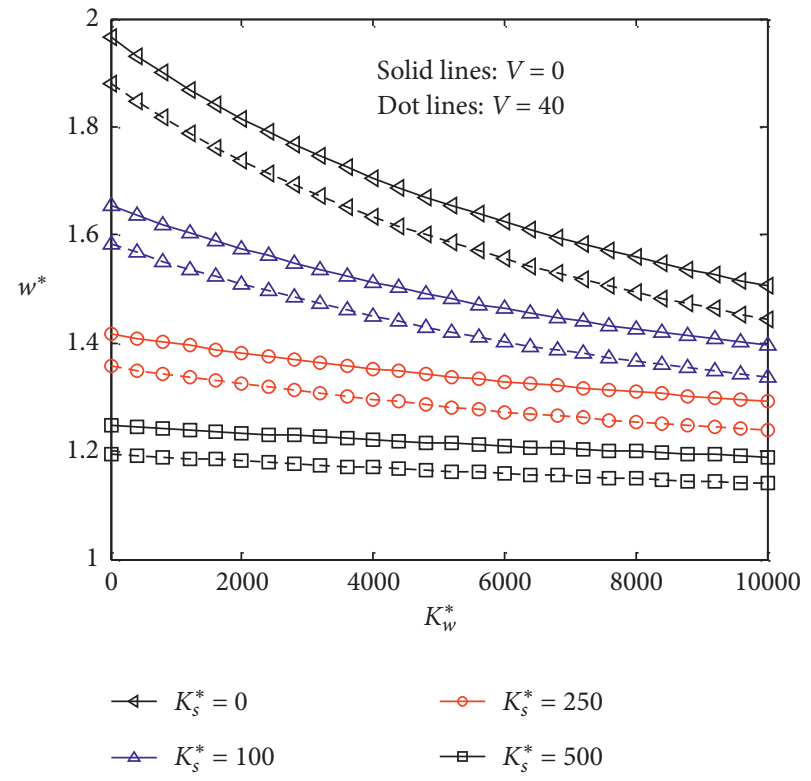

FIgURE 12: Deflection $w^{*}$ of the FGM plate under a uniformly distributed load of $10^{5} \mathrm{~N} / \mathrm{m}^{2}$ and actuator voltage $V=0$ and $40 \mathrm{~V}$, $n=1, K_{s}^{*}=0-500$ and $K_{w}^{*}=0-10000$.

$V=0 \mathrm{~V}$ and $V=40 \mathrm{~V}$ are plotted in Figures 15 and 16. It can be seen easily that when the area of the elastic foundation increases, the maximum deflection of the plate decreases; the reason is that when $d_{e}$ increases, the area of the elastic foundation increases, the total energy of the structure increases due to the additional energy of the foundation, then the plate becomes stronger, and the deflection of the plate decreases. Besides, when applying the piezoelectric effect to the plate, the maximum deflection of the plate decreases, too. 


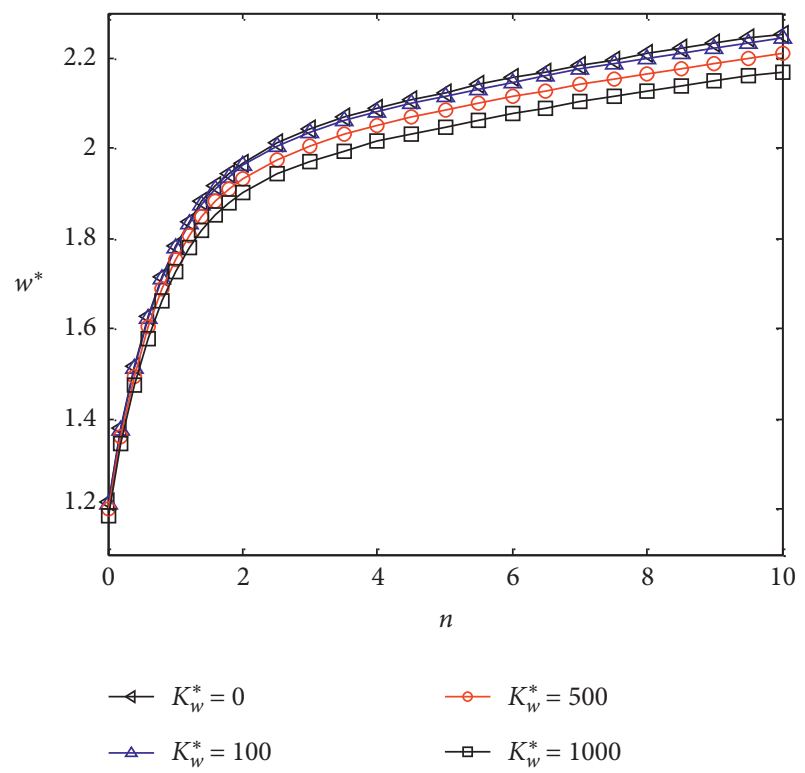

Figure 13: Deflection $w^{*}$ of the FGM plate under a uniformly distributed load of $10^{5} \mathrm{~N} / \mathrm{m}^{2}$ and actuator voltage $V=0 \mathrm{~V}, K_{s}^{*}=50$ and $K_{w}^{*}=0-1000$.

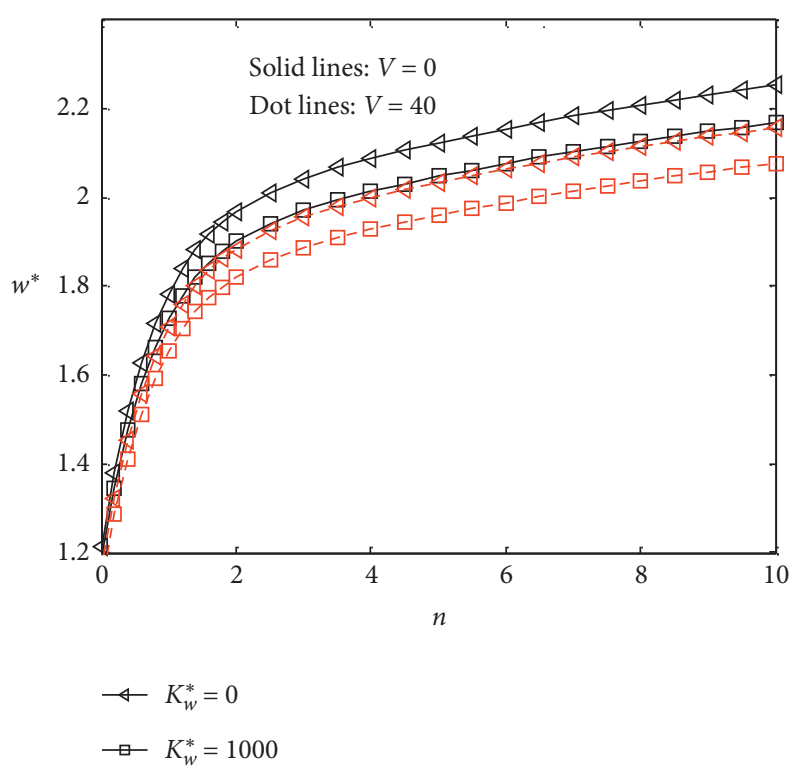

FIgURE 14: Deflection $w^{*}$ of the FGM plate under a uniformly distributed load of $10^{5} \mathrm{~N} / \mathrm{m}^{2}$ and actuator voltage $V=0$ and $40 \mathrm{~V}$, $K_{s}^{*}=50$ and $K_{w}^{*}=0-1000$.

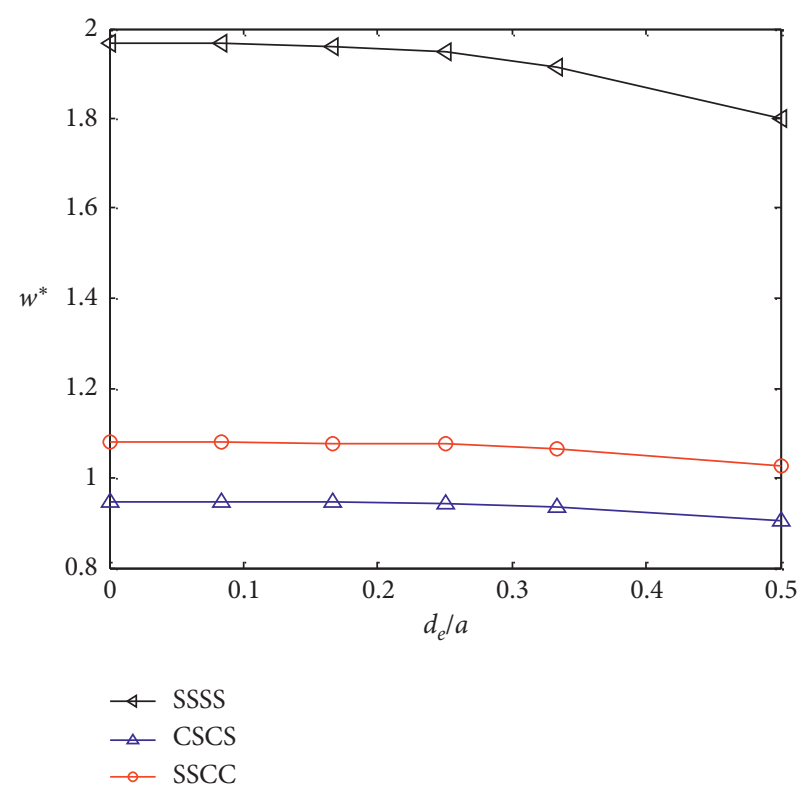

FIgURE 15: Deflection $w^{*}$ of the FGM plate under a uniformly distributed load of $10^{5} \mathrm{~N} / \mathrm{m}^{2}$ and actuator voltage $V=0, n=1$, $K_{s}^{*}=10$ and $K_{w}^{*}=100$.

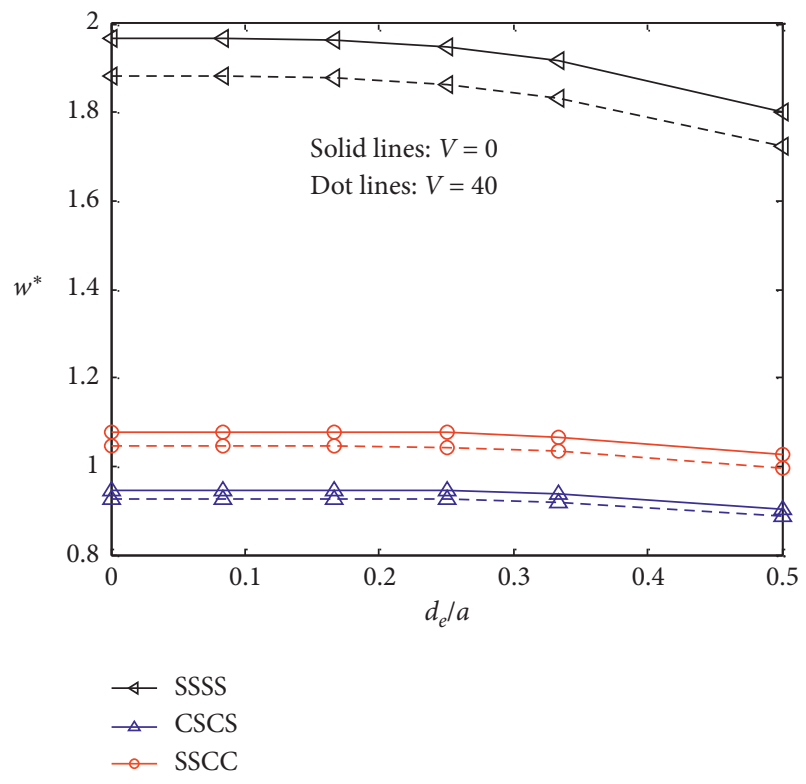

Figure 16: Deflection $w^{*}$ of the FGM plate under a uniformly distributed load of $10^{5} \mathrm{~N} / \mathrm{m}^{2}$ and actuator voltage $V=0$ and $40 \mathrm{~V}$, $n=1, K_{s}^{*}=10$ and $K_{w}^{*}=100$. 


\section{Conclusions}

This paper carries out the free vibration and static bending analysis of piezoelectric FGM plates resting on one area of the two-parameter elastic foundation. Finite element formulations are based on the third-order shear deformation theory of Reddy by using an 8-node plate element. The higher-order shear deformation theory of Reddy presents the advantages over the classical plate theory and the FSDT, especially the distribution of stresses in the thickness direction without the need for shear correction factors, which can solve free vibration, buckling, static, and dynamic behaviors of the plate made of various materials. The proposed theory and mathematical model are verified by comparing the numerical results of this work with those of exact solutions. The numerical comparisons show that, for the thirdorder shear deformation theory and the 8-node plate element used in this paper, the proposed approach has very good reliability; it can reasonably solve the problem of free oscillation and static bending of FGM plates with piezoelectric layers. Some key conclusions are summed up as follows:

(i) Increasing the stiffness of the elastic foundation, the nondimensional natural frequencies increase while the maximum deflections of the plate decrease. This can explain that the total energy of the plate is added by the energy of the foundation; therefore, the stiffness of the structure increases, the plate becomes stronger, the natural frequency of the plate increases, and the deflection of the plate decreases.

(ii) The larger the area of the elastic foundation $\left(d_{e}\right.$ increases), the higher the nondimensional natural frequencies, and the smaller the maximum deflections. This parameter also affects the free vibration mode shapes of the plate.

(iii) For each case of the elastic foundation, the corresponding volume fraction index $n$ can be found so that the nondimensional natural frequencies reach the smallest values.

(iv) When applying the piezoelectric effect to the plate, the maximum deflections of the structure decrease. This is the effect of the piezoelectric layers in the case of the plate under static load. This is due to the reason that the electrical force and mechanical force are in the opposite directions; therefore, the electrical force reduces the effect of the mechanical force, and the deflection of the plate decreases.

The numerical results in this work contribute to the knowledge of scientists when considering these types of piezoelectric structures in engineering practice.

\section{Data Availability}

The data used to support the findings of this study are included within the article.

\section{Conflicts of Interest}

The author declares that there are no conflicts of interest regarding the publication of this paper.

\section{Acknowledgments}

This work was supported by the Thuyloi University Foundation for Science and Technology Development (Grant no. 341(989)/QD-DHTL).

\section{References}

[1] S. S. Rao and M. Sunar, "Piezoelectricity and its use in disturbance sensing and control of flexible structures: a survey," Applied Mechanics Reviews, vol. 47, pp. 113-123, 1994.

[2] J. Tani, T. Takagi, and J. Qiu, "Intelligent material systems: application of functional materials," Applied Mechanics Reviews, vol. 51, pp. 505-521, 1998.

[3] E. Carrera, S. Brischetto, and P. Nali, Plates and Shells for Smart Structures, John Wiley \& Sons, Chichester, UK, 2011.

[4] M. Pohanka, "Overview of piezoelectric biosenzors, immunosensors and DNA sensors and their applications," Materials, vol. 11, p. 448, 2018.

[5] X. Wang, W. Zhou, Z. Wu, and X. Zhang, "Integrated design of laminated composite structures with piezocomposite actuators for active shape control," Composite Structures, vol. 215, pp. 166-177, 2019.

[6] A. Keshmiri, N. Wu, and Q. Wang, "A new nonlinearly tapered FGM piezolelectric energy harvester," Engineering Structures, vol. 173, pp. 52-60, 2018.

[7] N. D. Duc, P. H. Cong, and V. D. Quang, "Nonlinear dynamic and vibration analysis of piezo-electric eccenrically stiffened FGM plates in thermal environment," International Journal of Mechanical Sciences, vol. 115-116, pp. 711-722, 2016.

[8] B. A. Selim, L. W. Zhang, and K. M. Liew, "Active vibration control of FGM plates with piezoelectric layers based on Reddy's higher-order shear deformation theory," Composite Structures, vol. 155, pp. 118-134, 2016.

[9] X. L. Chen, Z. Y. Zhao, and K. M. Liew, "Stability of piezoelectric FGM rectangular plates subjected to nonuniformly distributed load, heat and voltage," Advances in Engineering Software, vol. 39, pp. 121-131, 2008.

[10] J. Maruani, I. Bruant, F. Pablo, and L. Gallimard, "A numerical efficiency study on the active vibration control for FGPM beam," Composite Structures, vol. 182, pp. 478-486, 2017.

[11] P. Sharma, "Efficacy of harmonic differential quadrature method to vibration analzsis of FGPM beam," Composite Structures, vol. 189, pp. 107-116, 2018.

[12] M. Komijani and R. Gracie, "Nonlienar thermo-electro-mechanical behaviour of FGPM beams," Composite Structures, vol. 150, pp. 208-218, 2016.

[13] S. Mikaeeli and B. Behjat, "Three-dimensional analysis of thick functionallz graded piezoelectric plate using EFG method," Composite Structures, vol. 154, pp. 591-599, 2016.

[14] Y. Q. Wang, "Electro-mechanical vibration analysis of functionally graded piezoelectric porous plates in the translation state," Acta Astronautica, vol. 143, pp. 263-271, 2018.

[15] B. Behjat, M. Salehi, A. Armin, M. Sadighi, and M. Abbasi, "Static and dynamic analysis of functionally graded piezoelectric plates under mechanical and electrical loading," Scientia Iranica, vol. 18, pp. 986-994, 2011. 
[16] Y. S. Li, J. H. Ren, and W. J. Feng, "Bending of sinusoidal functionally graded piezoelectric plate under in-plane megnetic field," Applied Mathematical Modelling, vol. 47, pp. 63-75, 2017.

[17] M. Adineh and M. Kadkhodayan, "Three-dimensional thermo-elastic analysis and dynamic response of a multidirectional functionally graded skew plate on elastic foundation," Composites Part B: Engineering, vol. 125, pp. 227-240, 2017.

[18] J. Yang and H. S. Shen, "Vibration characteristics and transient response of shear deformable functionally graded plates in thermal environments," Journal of Sound and Vibration, vol. 255, pp. 579-602, 2002.

[19] B. Q. Tinh, D. V. Thom, H. T. T. Lan et al., "On the high temperature mechanical behaviors analysis of heated functionally graded plates using FEM and a new third-order shear deformation plate theory," Composites Part B: Engineering, vol. 92, pp. 218-241, 2016.

[20] H. N. Nguyen, T. C. Tan, D. T. Luat, V. D. Phan, D. V. Thom, and P. V. Minh, "Research on the buckling behavior of functionally graded plates with stiffeners based on the thirdorder shear deformation theory," Materials, vol. 12, no. 8, p. 1262, 2019.

[21] V. H. Nam, H. D. Duc, M. K. Nguyen, V. D. Thom, and T. T. Hong, "Phase-field buckling analysis of cracked stiffened functionally graded plates," Composite Structures, vol. 217, pp. 50-59, 2019.

[22] Q. Li, Q. Wang, D. Wu, X. Chen, Y. Yu, and W. Gao, "Geometrically nonlinear dynamic analysis of organic solar cell resting on Winkler-Pasternak elastic foundation under thermal environment," Composites Part B: Engineering, vol. 163, pp. 121-129, 2019.

[23] Q. Li, D. Wu, W. Gao, F. Tin-Loi, Z. Liu, and J. Cheng, "Static bending and free vibration of organic solar cell resting on Winkler-Pasternak elastic foundation through the modified strain gradient theory," European Journal of Mechanics-A/ Solids, vol. 78, Article ID 103852, 2019.

[24] Y. Xiang, "Vibration of rectangular Mindlin plates resting on non-homogenous elastic foundations," International Journal of Mechanical Sciences, vol. 45, pp. 1229-1244, 2003.

[25] A. M. Zenkour, M. N. M. Allam, M. O. Shaker, and A. F. Radwan, "On the simple and mixed first-order theories for plates resting on elastic foundations," Acta Mechanica, vol. 220, pp. 33-46, 2011.

[26] A. M. Zenkour and A. F. Radwan, "On the simple and mixed first-order theories for functionally graded plates resting on elastic foundations," Meccanica, vol. 48, pp. 1501-1516, 2013.

[27] H. T. Thai and D. H. Choi, "A refined plate theory for functionally graded plates resting on elastic foundation," Composites Science and Technology, vol. 71, pp. 1850-1858, 2011.

[28] H. T. Thai, P. Minwo, and D. H. Choi, "A simple refined theory for bending, buckling, and vibration of thick plates resting on elastic foundation," International Journal of Mechanical Sciences, vol. 73, pp. 40-52, 2013.

[29] M. H. Yas and V. Tahouneh, "3-D free vibration analysis of thick functionally graded annular plates on Pasternak elastic foundation via differential quadrature method (DQM)," Acta Mechanica, vol. 223, pp. 43-62, 2012.

[30] H. S. Shen, "Nonlinear analysis of simply-supported ReissnerMindlin plates subjected to lateral pressure and thermal loading and resting on two-parameter elastic foundations," Engineering Structures, vol. 23, pp. 1481-1493, 2000.
[31] S. Chucheepsakul and B. Chinnaboon, "An alternative domain/boundary element technique for analyzing plates on two-parameter elastic foundations," Engineering Analysis with Boundary Elements, vol. 26, pp. 547-555, 2002.

[32] O. Civalek, "Nonlinear analysis of thin rectangular plates on Winkler-Pasternak elastic foundations by DSCHDQ methods," Applied Mathematical Modelling, vol. 31, pp. 606-624, 2007.

[33] H. S. Shen, J. Yang, and L. Zhang, "Free and forced vibration of Reissner-Mindlin plates with free edges resting on elastic foundations," Journal of Sound and Vibration, vol. 244, pp. 299-320, 2001.

[34] J. A. Abdalla and A. M. Ibrahim, "Development of a discrete Reissner-Mindlin element on Winkler foundation," Finite Elements in Analysis and Design, vol. 42, pp. 740-748, 2006.

[35] K. Ozgan and A. T. Daloglu, "Alternative plate finite elements for the analysis of thick plates on elastic foundations," Structural Engineering and Mechanics, vol. 26, pp. 69-86, 2007.

[36] A. M. Zenkour, "The refined sinusoidal theory for FGM plates on elastic foundations," International Journal of Mechanical Sciences, vol. 51, pp. 869-880, 2009.

[37] D. Zhou, Y. K. Cheung, S. H. Lo, and F. T. K. Au, “Threedimensional vibration analysis of rectangular thick plates on Pasternak foundation," International Journal for Numerical Methods in Engineering, vol. 59, pp. 1313-1334, 2004.

[38] A. M. Zenkour, "Benchmark trigonometric and 3-D elasticity solutions for an exponentially graded thick rectangular plate," Archive of Applied Mechanics, vol. 77, pp. 197-214, 2007.

[39] A. M. Zenkour, "Three-dimensional elasticity solutions for uniformly loaded cross-ply laminates and sandwich plates," Journal of Sandwich Structures \& Materials, vol. 9, pp. 213238, 2007.

[40] A. M. Zenkour, M. N. M. Allam, and M. Sobhy, "Bending of a fiber-reinforced viscoelastic composite plate resting on elastic foundations," Archive of Applied Mechanics, vol. 81, pp. 7796, 2011.

[41] N. D. Duc and P. H. Cong, "Nonlinear postbuckling of symmetric S-FGM plates resting on elastic foundations using higher order shear deformation plate theory in thermal environments," Composite Structures, vol. 100, pp. 566-574, 2013.

[42] A. Menasria, A. Bouhadra, A. Tounsi, A. A. Bousahla, and S. R. Mahmoud, "A new and simple HSDT for thermal stability analysis of FG sandwich plates," Steel \& Composite Structures, vol. 25, pp. 157-175, 2017.

[43] N. Mokhtar, A. H. Ait, B. Riadh, and T. Abdelouahed, "Vibration response and wave propagation in FG plates resting on elastic foundations using HSDT," Structural Engineering \& Mechanics, vol. 69, pp. 511-525, 2019.

[44] M. Abderrahmane, K. Abdelhakim, A. B. Abdelmoumen et al., "A four-unknown refined plate theory for dynamic analysis of FG-sandwich plates under various boundary conditions," Steel and Composite Structures, vol. 36, no. 3, pp. 355-367, 2010.

[45] Z. Abdallah, A. B. Abdelmoumen, B. Fouad et al., "Bending analysis of functionally graded porous plates via a refined shear deformation theory," Computers and Concrete, vol. 26, no. 1, pp. 63-74, 2020.

[46] R. Mohamed, H. B. Kouider, K. Abdelhakim et al., "A new innovative 3-unknowns HSDT for buckling and free vibration of exponentially graded sandwich plates resting on elastic foundations under various boundary conditions," Geomechanics and Engineering, vol. 22, no. 2, pp. 119-132, 2020. 
[47] K. Mokhtar, A. B. Abdelmoumen, K. Abdelhakim, B. Aicha, T. Abdelouahed, and S. R. Mahmoud, "Analytical modeling of bending and vibration of thick advanced composite plates using a four-variable quasi 3D HSDT," Engineering with Computers, vol. 36, pp. 807-821, 2020.

[48] C. C. Sara, K. Abdelhakim, A. B. Abdelmoumen et al., "A novel four-unknown integral model for buckling response of FG sandwich plates resting on elastic foundations under various boundary conditions using Galerkin's approach," Geomechanics and Engineering, vol. 21, no. 5, pp. 471-487, 2020.

[49] R. Salah, A. B. Abdelmoumen, B. Abdelhakim et al., "Effects of hygro-thermo-mechanical conditions on the buckling of FG sandwich plates resting on elastic foundations," Computers and Concrete, vol. 25, no. 4, pp. 311-325, 2020.

[50] C. R. Mohammed, A. Kaci, A. B. Abdelmoumen et al., "Influence of boundary conditions on the bending and free vibration behavior of FGM sandwich plates using a four-unknown refined integral plate theory," Computers and Concrete, vol. 25, no. 3, pp. 225-244, 2020.

[51] X. Q. He, T. Y. Ng, S. Sivashanker, and K. M. Liew, “Active control of FGM plates with integrated piezoelectric sensors and actuators," International Journal of Solids and Structures, vol. 38, pp. 1641-1655, 2001.

[52] J. N. Reddy, "Analysis of functionally graded plates," International Journal for Numerical Method in Engineering, vol. 47, pp. 663-684, 2000.

[53] M. Y. Yasin, N. Ahmad, and M. N. Alam, "Finite element analysis of actively controlled smart plate with patched actuators and sensors," Latin American Journal of Solids and Structures, vol. 7, pp. 227-247, 2010.

[54] S. Valliappan and K. Qi, "Finite element analysis of a smart damper for seismic structural control," Computers and Structures, vol. 81, pp. 1009-1017, 3003.

[55] H. S. Tzou and C. I. Tseng, "Distributed piezoelectric sensor/ actuator design for dynamic measurement/control of distributed parameter systems: a piezoelectric finite element approach," Journal of Sound and Vibration, vol. 138, pp. 17-34, 1990.

[56] M. A. Askari Farsangi and A. R. Saidi, "Levy type solution for free vibration analysis of functionally graded rectangular plates with piezoelectric layers," Smart Materials and Structures, vol. 21, Article ID 094017, 2012.

[57] S. Hosseini-Hashemi, H. Rokni Damavandi Taher, H. Akhavan, and M. Omidi, "Free vibration of functionally graded rectangular plates using first-order shear deformation plate theory," Applied Mathematical Modelling, vol. 34, pp. 1276-1291, 2010.

[58] J. B. Han and K. M. Liew, "Numerical differential quadrature method for Reissner/Mindlin plates on two-parameter foundations," International Journal of Mechanical Sciences, vol. 39, pp. 977-989, 1997. 